Cochrane Database of Systematic Reviews

\title{
Hyperbaric oxygen for carbon monoxide poisoning (Review)
}

Buckley NA, Juurlink DN, Isbister G, Bennett MH, Lavonas EJ

Buckley NA, Juurlink DN, Isbister G, Bennett MH, Lavonas EJ.

Hyperbaric oxygen for carbon monoxide poisoning.

Cochrane Database of Systematic Reviews 2011, Issue 4. Art. No.: CD002041.

DOI: 10.1002/14651858.CD002041.pub3.

www.cochranelibrary.com 
TABLE OF CONTENTS

HEADER 1

ABSTRACT

PLAIN LANGUAGE SUMMARY

SUMMARY OF FINDINGS

BACKGROUND

OBJECTIVES

METHODS

RESULTS

DISCUSSION

AUTHORS' CONCLUSIONS

ACKNOWLEDGEMENTS

REFERENCES

CHARACTERISTICS OF STUDIES

DATA AND ANALYSES

Analysis 1.1. Comparison 1 Hyperbaric Oxygen (HBO) vs. Normobaric Oxygen (NBO), Outcome 1 Presence of symptoms or signs at time of primary analysis (4-6 weeks).

APPENDICES

WHAT'S NEW

HISTORY

CONTRIBUTIONS OF AUTHORS

DECLARATIONS OF INTEREST

SOURCES OF SUPPORT

DIFFERENCES BETWEEN PROTOCOL AND REVIEW

NOTES

INDEX TERMS 
[Intervention Review]

\section{Hyperbaric oxygen for carbon monoxide poisoning}

Nick A Buckley ${ }^{1}$, David N Juurlink ${ }^{2}$, Geoff Isbister ${ }^{3}$, Michael H Bennett ${ }^{4}$, Eric J Lavonas 5

1Professorial Medicine Unit, POWH Clinical School, University of NSW, Randwick, Australia. ${ }^{2}$ Clinical Pharmacology and Toxicology, Sunnybrook Health Sciences Centre, University of Toronto, Toronto, Canada. ${ }^{3}$ Emergency Department, Newcastle Mater Misericordiae Hospital, Waratah, Australia. ${ }^{4}$ Department of Anaesthesia, Prince of Wales Hospital, Randwick, Australia. ${ }^{5}$ Rocky Mountain Poison and Drug Center, Denver Health and Hospital Authority, Denver, Colorado, USA

Contact address: Nick A Buckley, Professorial Medicine Unit, POWH Clinical School, University of NSW, South Wing, Edmund Blackett building, Prince of Wales Hospital, Randwick, NSW, 2031, Australia. n.buckley@unsw.edu.au.

Editorial group: Cochrane Injuries Group

Publication status and date: Edited (no change to conclusions), published in Issue 4, 2011.

Citation: Buckley NA, Juurlink DN, Isbister G, Bennett MH, Lavonas EJ. Hyperbaric oxygen for carbon monoxide poisoning. Cochrane Database of Systematic Reviews 2011, Issue 4. Art. No.: CD002041. DOI: 10.1002/14651858.CD002041.pub3.

Copyright @ 2011 The Cochrane Collaboration. Published by John Wiley \& Sons, Ltd.

\section{A B S T R A C T}

\section{Background}

Poisoning with carbon monoxide (CO) remains an important cause of accidental and intentional injury worldwide. Several unblinded nonrandomized trials have suggested that the use of hyperbaric oxygen (HBO) prevents the development of neurological sequelae. This has led to the widespread use of $\mathrm{HBO}$ in the management of patients with carbon monoxide poisoning.

\section{Objectives}

To examine randomised trials of the efficacy of hyperbaric oxygen (HBO) compared to normobaric oxygen (NBO) for the prevention of neurologic sequelae in patients with acute carbon monoxide poisoning.

\section{Search methods}

We searched the following electronic databases; Cochrane Injuries Group Specialised Register (searched June 2010), Cochrane Central Register of Controlled Trials (The Cochrane Library 2010, Issue 2), MEDLINE (Ovid SP) 1950 to June 2010, EMBASE (Ovid SP) 1980 to June 2010, ISI Web of Science: Science Citation Index Expanded (SCI-EXPANDED) 1970 to June 2010, ISI Web of Science: Conference Proceedings Citation Index-Science (CPCI-S) 1990 to June 2010.

\section{Selection criteria}

All randomised controlled trials of HBO compared to NBO, involving non-pregnant adults who are acutely poisoned with carbon monoxide (regardless of severity).

\section{Data collection and analysis}

Two authors independently extracted from each trial information on: the number of randomised patients, types of participants, the dose and duration of the intervention, and the prevalence of neurologic symptoms at follow-up.

\section{Main results}

Seven randomised controlled trials of varying quality were identified; one was excluded because it did not evaluate clinical outcomes. Of the six remaining trials involving 1361 participants, two found a beneficial effect of HBO for the reduction of neurologic sequelae at one month, while four others did not. One of these is an incomplete publication (an abstract of an interim analysis). Although pooled random effects meta-analysis does not suggest a significant benefit from HBOT (OR for neurological deficits $0.78,95 \% \mathrm{Cl} 0.54$ to 1.12 ), significant methodologic and statistical heterogeneity was apparent among the trials, and this result should be interpreted cautiously. Moreover, design or analysis flaws were evident in all trials. Importantly, the conclusions of one positive trial may have been influenced by failure to 
adjust for multiple hypothesis testing, while interpretation of the other positive trial is hampered by a high risk of bias introduced during the analysis including an apparent change in the primary outcome. Both were also stopped early 'for benefit', which is likely to have inflated the observed effect. In contrast three negative trials had low power to detect a benefit of HBO due to exclusion of severely poisoned patients in two and very poor follow-up in the other. One trial that was said to be finished around eight years ago has not reported the final analysis in any forum.

\section{Authors' conclusions}

Existing randomised trials do not establish whether the administration of $\mathrm{HBO}$ to patients with carbon monoxide poisoning reduces the incidence of adverse neurologic outcomes. Additional research is needed to better define the role, if any, of HBO in the treatment of patients with carbon monoxide poisoning. This research question is ideally suited to a multi-center randomised controlled trial.

\section{PLAIN LANGUAGE SUMMARY}

\section{There is insufficient evidence to support the use of hyperbaric oxygen for treatment of patients with carbon monoxide poisoning}

Many people are poisoned by carbon monoxide gas each year, either intentionally (e.g. in suicide attempts) or by accident. Carbon monoxide interferes with oxygen transport in the body, and can also directly damage a variety of organs including the brain. The usual treatment involves removing the affected person from the source of the gas, general supportive care, and administering oxygen which hastens the elimination of carbon monoxide from the body. High pressure oxygen (hyperbaric oxygen) is only available at a few hospitals, and it is sometimes used to speed this process even further. However, the review of published trials found conflicting, potentially biased, and generally weak evidence regarding the usefulness of hyperbaric oxygen for the prevention of neurological injury. 
SUMMARY OF FINDINGS

\section{Summary of findings for the main comparison. Hyperbaric Oxygen (HBO) compared to Normobaric Oxygen (NBO) for carbon monoxide poisoning}

Hyperbaric Oxygen (HBO) compared to Normobaric Oxygen (NBO) for carbon monoxide poisoning

Patient or population: patients with carbon monoxide poisoning

Settings: hospital

Intervention: Hyperbaric Oxygen (HBO)

Comparison: Normobaric Oxygen (NBO)

\begin{tabular}{|c|c|c|c|c|c|c|}
\hline \multirow[t]{3}{*}{ Outcomes } & \multicolumn{2}{|c|}{ Illustrative comparative risks ${ }^{\star}(95 \% \mathrm{Cl})$} & \multirow{3}{*}{$\begin{array}{l}\text { Relative ef- } \\
\text { fect } \\
(95 \% \mathrm{Cl})\end{array}$} & \multirow{3}{*}{$\begin{array}{l}\text { No of Partici- } \\
\text { pants } \\
\text { (studies) }\end{array}$} & \multirow{3}{*}{$\begin{array}{l}\text { Quality of the } \\
\text { evidence } \\
\text { (GRADE) }\end{array}$} & \multirow[t]{3}{*}{ Comments } \\
\hline & Assumed risk & Corresponding risk & & & & \\
\hline & Normobaric Oxygen (NBO) & Hyperbaric Oxygen (HBO) & & & & \\
\hline \multirow{4}{*}{$\begin{array}{l}\text { Presence of symptoms or } \\
\text { signs at time of primary } \\
\text { analysis (4-6 weeks) }\end{array}$} & Study population & & \multirow{4}{*}{$\begin{array}{l}\text { OR } \mathbf{0 . 7 8} \\
\text { (0.54 to } 1.12 \text { ) }\end{array}$} & \multirow{4}{*}{$\begin{array}{l}1361 \\
\text { (6 studies) }\end{array}$} & \multirow{4}{*}{$\begin{array}{l}\oplus \ominus \ominus \ominus \\
\text { very low } 1,2,3,4,5\end{array}$} & \\
\hline & 334 per 1000 & $\begin{array}{l}\mathbf{2 8 1} \text { per } \mathbf{1 0 0 0} \\
\text { (213 to } 360)\end{array}$ & & & & \\
\hline & \multicolumn{2}{|l|}{ Medium risk population } & & & & \\
\hline & 338 per 1000 & $\begin{array}{l}\mathbf{2 8 5} \text { per } \mathbf{1 0 0 0} \\
\text { (216 to } 364 \text { ) }\end{array}$ & & & & \\
\hline
\end{tabular}

${ }^{\star}$ The basis for the assumed risk (e.g. the median control group risk across studies) is provided in footnotes. The corresponding risk (and its $95 \%$ confidence interval) is based on the assumed risk in the comparison group and the relative effect of the intervention (and its $95 \% \mathrm{Cl}$ ).

Cl: Confidence interval; OR: Odds ratio;

GRADE Working Group grades of evidence

High quality: Further research is very unlikely to change our confidence in the estimate of effect.

Moderate quality: Further research is likely to have an important impact on our confidence in the estimate of effect and may change the estimate.

Low quality: Further research is very likely to have an important impact on our confidence in the estimate of effect and is likely to change the estimate.

Very low quality: We are very uncertain about the estimate.

1 All studies had high risk of bias for various reasons. Most were unblinded and used self-reported outcomes, follow-up was sometimes poor, and most RCTs terminated prematurely.

2 Two RCTS show extremely large benefit and four no benefit. Statistical measures indicate a high degree of heterogeneity.

3 The numbers of severe long-term clinical outcomes in the RCTS was very low and not always reported. Most studies focussed on outcomes determined largely or entirely on non-specific self-reported symptoms.

4 The overall pooled estimate confidence interval and individual RCT reports are consistent with both benefit and harm resulting from HBO. 


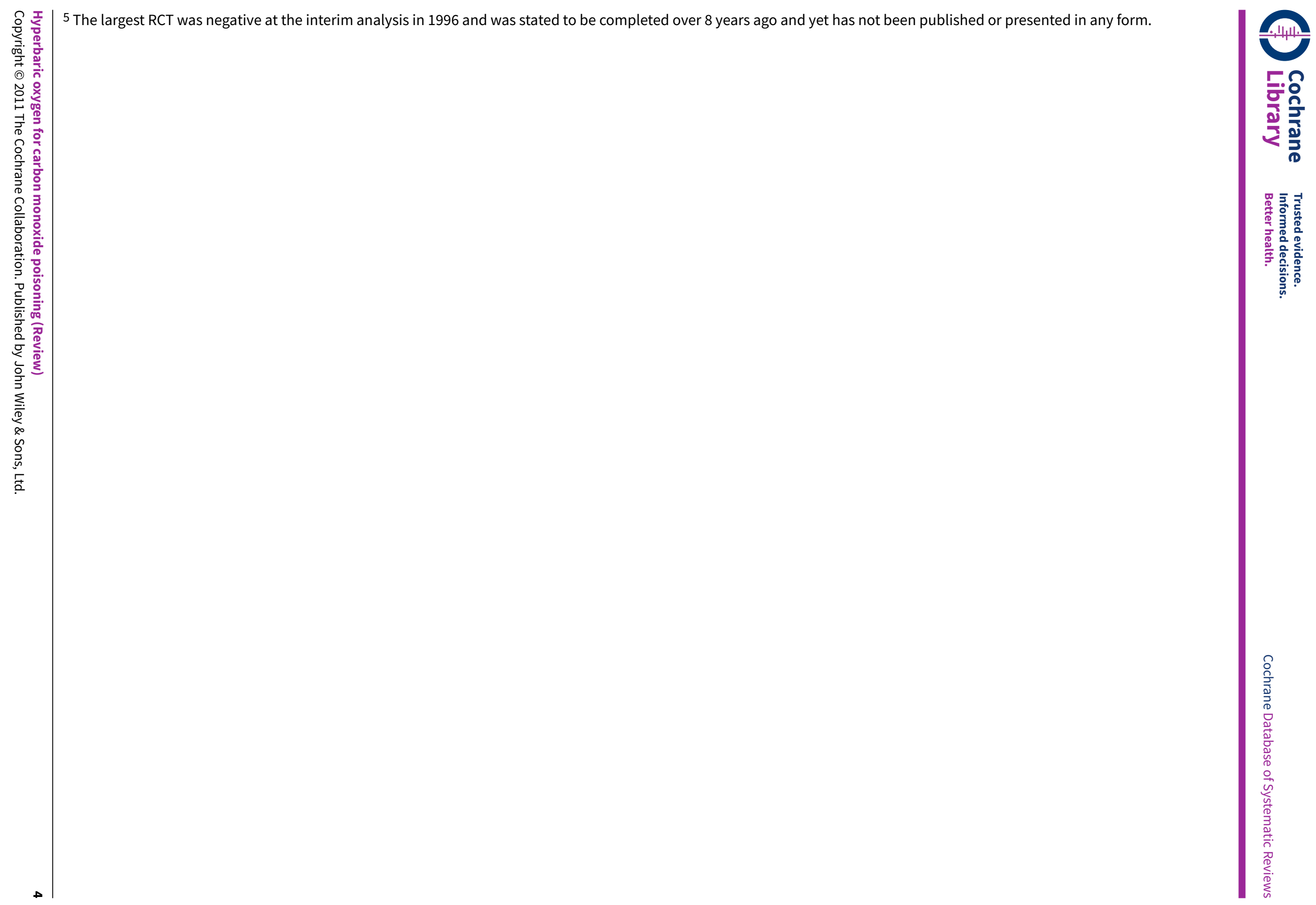




\section{B A C K G R O U N D}

Carbon monoxide ( $\mathrm{CO}$ ) is a colourless, odourless and tasteless gas generated during the incomplete combustion of carbon-based compounds. Poisoning with CO remains an important cause of accidental and intentional injury worldwide. In the United States alone, there are an estimated 1,000-2,000 accidental deaths due to carbon monoxide exposure each year, resulting from an estimated 50,000 annual exposures (Hampson 2007).

\section{Description of the condition}

The pathophysiology of carbon monoxide poisoning is incompletely understood. Upon exposure, CO binds to haemoglobin with an affinity 210 times than of oxygen and also increases the affinity of the remaining sites for oxygen. Thus it decreases both the oxygen-carrying and oxygen-delivery capacity of blood. In addition to generating carboxyhaemoglobin, carbon monoxide has been shown to lead to harm by several other mechanisms including direct disruption of cellular oxidative processes by binding to myoglobin and cytochromes, and during recovery there is marked oxidative stress and inflammatory responses (Weaver 2009). Both the direct hypoxic effects and the subsequent oxidative stress and inflammatory processes lead to varying degrees of end-organ damage, and occasionally death. The severity of poisoning is a function of the duration of exposure and the ambient concentration of $\mathrm{CO}$, and the underlying health status of the exposed individual. Although useful for diagnosis when detected, the initial carboxyhaemoglobin level correlates poorly with outcome (Hampson 2008; Weaver 2007).

Two syndromes are recognized to occur after acute $\mathrm{CO}$ poisoning: persistent neurologic sequelae (PNS) and delayed neurologic sequelae (DNS) (Weaver 1999; Weaver 2009). The former is characterized by symptoms or signs attributable to $\mathrm{CO}$ poisoning that are evident immediately following poisoning. These may improve, although not always to the premorbid state. The latter is characterized by the development of new symptoms or signs referable to $\mathrm{CO}$ poisoning, occurring after a period of days to weeks. This deterioration may be abrupt and dramatic. In both instances, however, the signs and symptoms can be non-specific, and the entities may be difficult to distinguish from each other. The symptoms and signs range from subtle personality changes, mood disorders and memory loss to (much less commonly) focal neurological injuries and other severely disabling manifestations of hypoxic brain injury. The definitions for this outcome in clinical studies have varied considerably.

\section{Description of the intervention}

Hyperbaric oxygen (HBO) refers to the administration of $100 \%$ oxygen at pressures higher than atmospheric pressure. This is performed in a hyperbaric chamber. However, there are a very wide range of protocols used in the treatment of carbon monoxide poisoning involving different pressures, durations, and repeated treatments (Hampson 2006), and this variation is also apparent in the RCTs in this review.

\section{How the intervention might work}

Standard treatment for $\mathrm{CO}$ poisoning includes removal from the site of exposure, administration of supplemental oxygen, and general supportive care (Wolf 2008). The elimination halflife of carboxyhaemoglobin (approximately 320 minutes in room air) is shortened approximately five-fold by the administration of $100 \%$ oxygen at atmospheric pressure (normobaric oxygen, NBO). The administration of HBO further hastens the elimination of carboxyhaemoglobin (Jay 1997). Animal studies have demonstrated benefits of $\mathrm{HBO}$ on a range of parameters including learning and memory (e.g. Thom 2006); although other animal studies have shown equal effects from NBO and HBO on such parameters (e.g. Bunc 2006; Gilmer 2002).

\section{Why it is important to do this review}

Several unblinded, non-randomised trials and case series suggested that the use of HBO prevents the development of PNS and/or DNS. These observations led some clinicians to use HBO for selected patients with carbon monoxide poisoning, although there is considerable variability in clinical practice. Because HBO is available at only a few hospitals (necessitating the transfer of potentially unstable patients), is substantially more expensive than NBO, and is occasionally complicated by barotrauma (Weaver 2009), seizures (Sanders 2009), pulmonary edema (Weaver 2001) and claustrophobia, its superiority over NBO in this setting should be established (Wolf 2008). This review was undertaken to examine the effect of $\mathrm{HBO}$ on the development of neurologic symptoms due to $\mathrm{CO}$ poisoning one month after treatment.

\section{O B JECT IVES}

To examine the efficacy of $\mathrm{HBO}$ in reducing the prevalence of neurologic signs and symptoms approximately 4 to 6 weeks following treatment in patients with acute $\mathrm{CO}$ poisoning.

\section{METHODS}

\section{Criteria for considering studies for this review}

\section{Types of studies}

The analysis was limited to randomised controlled trials, with or without blinding. Trials that used surrogate outcome measures, did not report a frequency of neurologic sequelae, or did not present data allowing the calculation of the frequency of neurologic symptoms at one month were excluded from the analysis.

\section{Types of participants}

All non-pregnant adults acutely poisoned by carbon monoxide.

\section{Types of interventions}

Studies were included in which patients with $\mathrm{CO}$ poisoning were randomised to receive either $\mathrm{HBO}$ or NBO.

\section{Types of outcome measures}

The main outcome measure of interest was the presence of signs or symptoms possibly indicative of neurologic injury at follow-up (approximately 4 to 6 weeks) after randomisation. No universally accepted criteria exist for the diagnosis of persistent neurologic sequelae (PNS) or delayed neurologic sequelae (DNS) and outcomes were defined and measured differently in each study. In the absence of a well-defined, mutual outcome measure for all studies, we retained the definitions of neurologic sequelae presented by investigators in their reports.

The signs and symptoms were often non-specific and included headache, confusion, difficulty concentrating, and sleep 
disturbances, ataxia, and difficulty with memory and executive processing, all of which are plausibly related to $\mathrm{CO}$ exposure. All studies simply reported neurological sequelae as present or absent at specified times of follow-up. While it is possible that HBO may also reduce the severity of neurologic sequelae among affected persons, no trials formally compared severity ratings or other nondichotomised measures of function between NBO and HBO-treated patients.

\section{Search methods for identification of studies}

The searches were not restricted by date, language or publication status.

\section{Electronic searches}

We searched the following electronic databases;

- Cochrane Injuries Group Specialised Register (searched 07 June 2010),

- Cochrane Central Register of Controlled Trials (The Cochrane Library 2010, Issue 2),

- MEDLINE (Ovid SP) 1950 to May Week 4 2010,

- EMBASE (Ovid SP) 1980 to Week 22 (June) 2010,

- PubMed [www.ncbi.nlm.nih.gov/sites/entrez/] (searched 9 June 2010 (added to PubMed in the last 180 days)),

- ISI Web of Science: Science Citation Index Expanded (SCIEXPANDED) 1970 to June 2010,

- ISI Web of Science: Conference Proceedings Citation IndexScience (CPCI-S) 1990 to June 2010.

The electronic search strategies were formulated and run by the Cochrane Injuries Group's Trials Search Co-ordinator. The search strategies are reported in full in Appendix 1.

\section{Searching other resources}

Additional references were sought from experts who have published widely in the area. We also examined the reference lists of relevant papers identified by the search as well as other published reviews. We also searched the meta-register of controlled trials for trial protocols (www.controlled-trials.com) - [searched June 2010].

\section{Data collection and analysis}

The Cochrane Injuries Group Trials Search Co-ordinator ran the searches and collated the search results, using bibliographic software so that duplicates could be removed before passing the results on to the review authors.

\section{Selection of studies}

Two authors (DNJ, NAB) screened the electronic search results for possibly relevant trials and these articles were retrieved in full. An additional abstract (Mathieu 1996) was identified in a manual search of the reference lists of the other studies. We also identified abstracts of the interim analyses of three trials (Scheinkestel 1999; Thom 1995; Weaver 2002) as well as reports that included analyses of other outcomes from one trial (Weaver 2002; Weaver 2005). Several trials of HBO with objectives other than treatment of carbon monoxide poisoning were excluded without further evaluation. Three RCTs which gave $\mathrm{HBO}$ to all patients were also excluded (Gao 2002; Hampson 2006; Weaver 2007). Seven potentially relevant trials were identified. One trial was excluded because of inadequate allocation concealment and absence of any recorded clinical outcomes (Ducasse 1995).

\section{Data extraction and management}

Two reviewers (DNJ, NAB) extracted data from each trial including information on the participants (age and gender distribution, mode of poisoning, carboxyhaemoglobin level upon randomisation, and history of loss of consciousness), the interventions (duration and dose of NBO and $\mathrm{HBO}$ ), and presence of signs and symptoms at follow-up. Insufficient data were available to examine the effect of $\mathrm{HBO}$ in any subgroup of patients.

\section{Assessment of risk of bias in included studies}

The methods used in the review have been updated since publication of the protocol, to reflect revised guidance from The Cochrane Collaboration. The following items were assessed using the risk of bias assessment tool (Higgins 2008).

-Was there adequate sequence generation?

-Was allocation adequately concealed?

- Was knowledge of the allocated interventions adequately prevented during the study?

- Were incomplete outcome data adequately addressed?

- Are reports of the study free of suggestion of selective outcome reporting?

-Was the study apparently free of other problems that could put it at a risk of bias?

Two authors (EL, MHB) independently assessed the risk of bias of each included study against these criteria. Studies that did not adequately address these criteria were considered at high risk of bias. Authors were not blinded with respect to study authors, institution, or journal. The authors resolved disagreements by consensus, with a third author (NB) to be consulted if disagreements persisted.

Where the method of allocation concealment was not reported, or where additional information was required to appropriately assess study quality, the principal authors of these trials were contacted for clarification. We contacted the authors of four studies and received two replies; however, the responses did not uniformly clarify our questions.

\section{Measures of treatment effect}

All trials reported dichotomised outcomes (normal/sequelae) although the definition of these varied between trials. For the purpose of pooled analysis these were converted to odds ratios. No study reported results comparing the severity of neurological sequelae among affected patients in the NBO and HBO groups.

\section{Assessment of heterogeneity}

The trials are extremely heterogenous in terms of their settings, types of participants, severity of poisoning, interventions in $\mathrm{HBO}$ and NBO arms and outcome measure definitions (see Characteristics of included studies). [Formal statistical assessment of heterogeneity was not possible due to the small number of studies.] For this reason a random (rather than fixed) effects model was applied to the pooled analysis. 


\section{RES U L T S}

\section{Description of studies}

Of the six trials included, five are published in final form (Annane 2010; Raphael 1989; Scheinkestel 1999; Thom 1995; Weaver 2002). One has only been published as an abstract of an interim analysis (Mathieu 1996), is not registered, and the lead author has not responded to repeated requests for further information. Data from all six trials are incorporated in this review, although it is possible that more details regarding this last trial may become available when it is published in final form. The extensive delay to date raises questions as to whether this trial will ever be published.

The diagnosis of carbon monoxide poisoning was generally made by a history of exposure to carbon monoxide and an elevated carboxyhaemoglobin level. The severity of $\mathrm{CO}$ poisoning varied in the individual trials. Two studies stratified randomisation according to whether or not there was a history of loss of consciousness (Annane 2010; Raphael 1989). In the earlier of these (Raphael 1989), only patients with no initial loss of consciousness were randomised to HBO vs. NBO, while in the latter (Annane 2010), patients with transient loss of consciousness were randomised to $\mathrm{HBO}$ vs. NBO. In both of these studies, patients with more marked impairment of consciousness were randomised to different doses of HBO; these patients were not considered in this review. Two trials only enrolled patients with no loss of consciousness or cardiovascular instability (Mathieu 1996; Thom 1995), while two other trials included all patients with $\mathrm{CO}$ poisoning regardless of severity (Scheinkestel 1999; Weaver 2002).

Each principal investigator confirmed that virtually all patients received treatment with supplemental oxygen prior to randomisation, which would be considered standard practice.

The patients, interventions, completeness of follow-up and outcomes assessment varied significantly among the trials (see section on included studies for full details). Only two trials (Scheinkestel 1999; Weaver 2002) employed 'sham dives', exposing control subjects to NBO in a hyperbaric chamber.

The duration, timing, and dose of both $\mathrm{HBO}$ and NBO also varied greatly among studies. Two studies from the same unit (Annane 2010; Raphael 1989) used an active treatment of HBO at 2 atmospheres absolute (ATA) for two hours followed by $100 \%$ oxygen at atmospheric pressure for four hours, while the control group received $100 \%$ oxygen for six hours. Another trial (Thom 1995) varied the intensity of $\mathrm{HBO}$ in the active treatment arm, using 2.8 ATA for 0.5 hours followed by 2 ATA for 1.5 hours. In this trial the control group received $100 \%$ oxygen at 1 ATA for a variable duration (mean $=4.2$ hours). A trial published thus far only as an interim analysis (Mathieu 1996), randomised patients to HBO at 2.5 ATA for 90 minutes or NBO for 12 hours. In another trial (Weaver 2002), patients in the active treatment arm received three sessions of $\mathrm{HBO}$, including an initial treatment of 3 ATA for 1 hour followed by 2 ATA for 1 hour, with two additional 2-hour treatments of 2 ATA at 6-12 hour intervals. Controls underwent sham dives with NBO at 1 ATA.

The intervention in one trial (Scheinkestel 1999) was unusual and substantially different from other studies. Patients randomised to active treatment received $\mathrm{HBO}$ at 2.8 ATA for 60 minutes followed by $100 \%$ oxygen by face mask for 40 minutes, but were then administered high-flow oxygen for the remainder of the day. This sequence was repeated daily for a total of three days. A further $28 \%$ received three additional treatments because they were deemed to have a poor outcome. The control group was treated with $100 \%$ NBO for 100 minutes followed by high-flow oxygen, repeated daily for three days. Thereafter, $15 \%$ underwent the same three-day regimen repeated because they were felt to have a poor outcome.

\section{Risk of bias in included studies}

All included trials were at considerable risk of bias (see Characteristics of included studies). One study had very limited reporting of methods as it was published only as an abstract (Mathieu 1996). It is unclear why this negative study (at the interim analysis) has never been published in full but the risk of bias from this study must be judged to be consequently very high. Four studies including this one had minimal or no blinding (Annane 2010; Mathieu 1996, Raphael 1989; Thom 1995) and all participants and study personnel were probably aware of allocation. In these trials double-blinding was not possible as the control patients did not enter a hyperbaric chamber and the possibility of performance, attrition or detection bias is particularly strong as the main outcomes were the patient's reports of new symptoms.

Only two trials (Weaver 2002; Scheinkestel 1999) stipulated that patients and outcome assessors were blind to treatment allocation. This was facilitated by sham treatments in the hyperbaric chamber. However these studies had other factors that indicated there was still a high risk of bias. Even for these blinded studies, it would have been relatively easy for those conducting the analysis to identify treatment arms in light of specific adverse events seen with hyperbaric therapy, which would be much more common in that treatment arm of the trials. It appears that a large risk of bias was introduced during analysis in both these trials. In one trial (Weaver 2002), numerous assumptions were applied, all of which favour HBO. These include well documented changes in the primary outcome as well as the diagnostic thresholds for their definition of neurologic sequelae (Buckley 2005; Buckley 2005a). A detailed exposition of these is available in the Risk of bias tables in Characteristics of included studies. In the other blinded study, multiple analyses were applied without statistical adjustment reaching a tenuous conclusion that HBO increased the rate of DNS in one sub-group (Scheinkestel 1999). Each of the studies published in full adequately outlined the numbers of patients lost to followup and reasons for this. However, follow-up was particularly poor in this negative study (Scheinkestel 1999), in which only $46 \%$ of subjects were evaluated one month after treatment.

For further explanations and detailed trial quotes see Risk of bias tables in Characteristics of included studies.

\section{Effects of interventions}

See: Summary of findings for the main comparison Hyperbaric Oxygen (HBO) compared to Normobaric Oxygen (NBO) for carbon monoxide poisoning

The six trials enrolled a total of 1997 patients, of whom 1335 were randomised to either $\mathrm{HBO}$ or NBO and had outcomes recorded. The severity of $\mathrm{CO}$ poisoning, the treatment regimens, and outcome assessment varied significantly among trials.

The prevalence of persistent signs or symptoms of CO poisoning (as defined by the investigators) at 4 to 6 weeks following treatment was 202 of $691(29 \%)$ patients treated with HBO, compared with 
219 of $644(34 \%)$ patients treated with NBO. In a pooled analysis using a random effects model, no statistically significant reduction in neurologic sequelae was associated with HBO treatment (OR $0.78 ; 95 \%$ confidence interval 0.54 to 1.12 ) (Analysis 1.1), although the point estimate favoured treatment. Simple pooling of trial results with a confidence interval should not be taken to be a true estimate of the predicted effects of treatment expected in future studies or individuals (Riley 2011). The methodologic and statistical heterogeneity among the various trials and the high risk of bias in all studies even further increase the uncertainty around any estimate, well beyond that provided by statistical techniques.

Sub-group analyses of specific groups of patients (e.g. those with more severe poisoning, those with deliberate self-poisoning, or those who received treatment relatively early) was not possible because individual patient characteristics and outcomes could not be determined from each study.

Detailed review of the trials and, where available, earlier published abstracts of interim analyses, identified several aspects that warrant interpretive caution with regard to the reported effect of the interventions in individual studies (also see Characteristics of included studies):

\section{Raphael 1989 \& Annane 2010}

These trials found no benefit from HBO over NBO, but the investigators only permitted less severely poisoned patients to be randomised to $\mathrm{HBO}$ or NBO. Because interventions are, in general, most likely to show benefit in patients with more severe disease, the possibility of type II error in these trials is high. Consequently, this trial does not disprove a benefit of $\mathrm{HBO}$, particularly in more severely poisoned patients.

\section{Thom 1995}

This study was the first published RCT to claim a benefit from the use of $\mathrm{HBO}$, however it was very small and outcome assessment was performed by unblinded clinicians. The statistical analysis raises several issues that all substantially increase the likelihood of a type I error. These include a failure to adjust the level of significance for an interim analysis and multiple comparisons, implausibly high levels of statistical significance $(\mathrm{P}<0.005)$ presented at the interim analysis, and the trial's seemingly premature termination based upon the main analysis. (Although no sample size calculation was reported, no power calculation would have led to the design of such a small trial for this outcome.)

\section{Mathieu 1996}

This trial is reported only as an interim analysis. Although no statistically significant difference in neurologic symptoms was found between treatment arms at 1 month (26\% with NBO vs. $22 \%$ with HBO), a difference was reported at 3 months (15\% vs. $9 \% ; \mathrm{P}=0.016)$. No significant difference was reported at 6 months follow-up ( $10 \%$ vs. $6 \%$ respectively; $P=0.09)$ or 1 year $(5 \%$ vs. $4 \%$ respectively), although the final results of this trial have yet to be reported in any forum. Of note, this trial is sometimes characterized as demonstrating a benefit to HBO (Hampson 2004; Thom 2002) based solely upon the interim analysis findings at 3 months, despite a lack of adjustment for multiple hypothesis testing and the absence of a significant difference at other intervals.

Scheinkestel 1999
This is the only negative study published to date in which control patients received sham treatment in a hyperbaric chamber. The trial enrolled a relatively large number of patients with attempted suicide, and patients in both arms of the trial were treated with continuous normobaric oxygen for 3 days, which is not generally accepted as standard practice. The most serious threat to the interpretation of this study is that only $46 \%$ of patients randomised to treatment were followed up at 4 to 6 weeks. Because of the strong possibility that patients lost to follow-up were systematically different from those in whom follow-up testing was obtained, the results of this trial are difficult to interpret.

Weaver 2002

This is the only positive study published to date in which control patients received sham treatment in a hyperbaric chamber. While the technical aspects of the conduct of this trial (e.g. blinding, randomisation, allocation concealment, high followup) are clearly superior to previously published trials and the effect size of HBO appears large, this trial's interpretation is hampered by the high risk of bias introduced during analysis. First, although the final publication (Weaver 2002) describes a primary outcome of all neurologic sequelae, the originally intended endpoint was delayed neurologic sequelae (Weaver 1995) and subsequent reports indicate there was no significant difference for this outcome (Weaver 2005). Second, the thresholds for the definition of neurologic sequelae itself changed between the interim analysis (Weaver 1995) and the final publication (Weaver 2002), in which non-specific symptoms were the primary determinant of a statistical difference between treatments. In the final publication (Weaver 2002), neuropsychological testing identified no difference between $\mathrm{HBO}$ and NBO; indeed, the mean neuropsychological testing scores for patients treated with NBO were within the normal range. It is probable that additional bias was introduced in the analysis from the drop-out and last observation carried forward assumptions for 6, 26 and 52 week outcomes and the premature termination of the trial "for benefit", all of which increased estimates of the magnitude of benefit for HBO. [Note that although the interim and efficacy analyses were performed blind to the assignment, the very different chamberrelated adverse event rates in the two arms suggest that anyone with access to the comparison of the rates of these outcomes would have been able to identify treatment allocation.]

\section{DISCUSSION}

This systematic review identified all published randomised controlled trials of $\mathrm{HBO}$ vs. NBO for the treatment of acute carbon monoxide poisoning. The six included trials enrolled patients with carbon monoxide poisoning of varying severity, employed different regimens of hyperbaric and normobaric oxygen, had varying degrees of follow-up, and were subject to various biases and analytical flaws that may have substantially influenced their conclusions. Only two of these trials (Scheinkestel 1999 and Weaver 2002) were conducted in a double-blind fashion, and none reported clinically significant long-term outcomes. HBO has potential to cause harm as well as benefit. This includes the expected and well-known common bariatric and hyperoxic adverse effects. The significantly worse outcomes with 2 vs. 1 HBO treatments (Annane 2010 - Trial B (excluded from this review)) suggests the possibility that in some circumstances HBO might even worsen the risk of neurological sequelae. 
Although we present a pooled analysis of these six studies suggesting no statistically significant difference between $\mathrm{HBO}$ and NBO (OR 0.78; 95\% confidence interval 0.54 to 1.12 ), we caution that the methodologic and statistical heterogeneity of the six trials renders this analysis difficult to interpret (Riley 2011). Subanalyses by severity, intent, and duration of poisoning were not possible. The overall non-significant difference is not dependent on the data from any single study, a meta-analysis of any 5 of the studies or just the 5 published in full remains non-significant.

During the course of this review we also identified several publications authored by recognized content experts. These often promoted the use of HBO based on the results of the two positive trials (Weaver 2002) and, occasionally, the 3-month interim analysis of Mathieu's study (Hampson 2004; Mathieu 1996; Thom 2002) without addressing the limitations set forth above. We acknowledge that many in the hyperbaric medicine community strongly advocate the position that HBO has been established as effective (e.g. Stoller 2007, Weaver 2009, http://www.cochranefeedback.com/cf/cda/ citation.do?id=9585\#9585; http://www.cochranefeedback.com/cf/ cda/citation.do?id=9569\#9569; Logue 2008), and that further placebo-controlled studies are therefore unethical. Other groups disagree with that position, explicitly (Wolf 2008; Morrison 2010) or implicitly (Vanden Hoek 2010) calling for additional clinical trials. Based on the extreme heterogeneity of results from published clinical trials and the significant limitations of these trials, as detailed above, we believe that additional placebo-controlled clinical trials of $\mathrm{HBO}$ for the treatment of $\mathrm{CO}$ poisoning are ethical, warranted, and necessary. It should be noted that, at present, no registered trials meeting our inclusion criteria are currently in progress.

\section{AUTHORS' CONCLUSIONS}

\section{Implications for practice}

Existing randomised controlled trials of $\mathrm{HBO}$ vs. NBO in the treatment of non-pregnant adults with acute carbon monoxide poisoning provide conflicting results regarding the efficacy of HBO. All published studies have limitations that threaten and may invalidate their conclusions.

Based on the results of these trials, HBO cannot be routinely recommended for the treatment of $\mathrm{CO}$ poisoning. It is possible that some patients, particularly those with more severe poisoning, may derive benefit from treatment, but this remains unproven.

\section{Implications for research}

Additional research is needed to further define the role, if any, of hyperbaric oxygen in the treatment of carbon monoxide poisoning in various subsets of patients. Because carbon monoxide poisoning occurs commonly in diverse settings, this research is ideally suited to a multi-centre randomised trial. Given the heterogeneity of results in the published trials, we believe equipoise for such a trial exists.

In order to minimize bias, future studies should employ a tripleblind technique (investigators, patients, and outcome assessors) using sham dives in a hyperbaric chamber for control subjects (as in Weaver 2002 and Scheinkestel 1999). However, in order to minimise the impact of any inadvertent unblinding during analysis, the trial should be prospectively registered with explicit definitions of primary and secondary outcomes and monitored in an ongoing fashion by an independent data monitoring committee.

The use of meaningful clinical outcomes is encouraged, while multiple hypothesis testing should be minimized unless investigators make appropriate statistical adjustments to minimize inflation of the type I error rate. Stratified analyses of several clinically important patient subgroups (including those with deliberate self-poisoning, impaired consciousness, and those treated shortly after exposure) should be conducted; these should also be pre-specified.

Importantly, given the absence of reliable evidence that patients with severe $\mathrm{CO}$ poisoning benefit from HBO therapy, exclusion of such patients from future trials is not justified. Indeed, the potential to demonstrate any effects of treatment is greatest in this population, because they are at the highest risk of adverse outcomes (Sackett 2001).

\section{ACKNOWLEDGEMENTS}

We thank Mike Stanbrook, Michael McGuigan \& Barry Stokes who contributed to earlier versions of this review. We are also grateful to Karen Blackhall (from the Cochrane Injuries Group) who helped with updating the search; and Emma Sydenham (Injuries Group) and Miranda Cumpston (Australian Cochrane Centre) for other assistance in revising this review in line with the updated guidance documents. Thanks also to Taixiang Wu from the Chinese Cochrane Centre who helped us to obtain a copy of a Chinese RCT (subsequently excluded). 


\section{RE F E R E N C E S}

\section{References to studies included in this review}

Annane 2010 \{published and unpublished data\}

* Annane D, Chadda K, Gajdos P, Jars-Guincestre M-C, Chevret S, Raphael J-C. Hyperbaric oxygen therapy for acute domestic carbon monoxide poisoning: two randomized controlled trials. Intensive Care Medicine 2010;ePub (in press):DOI 10.1007/ s00134-010-2093-0.

Annane D, Raphael JC. Hyperbaric Oxygen Therapy for Comatose Patients With Acute Carbon Monoxide Poisoning. clinical trials.gov April 7 2010. [NCT01099995]

Raphael JC, Chevret S, Driheme A, Annane D. Managing carbon monoxide poisoning with hyperbaric oxygen (Abstract). Journal of Toxicology - Clinical Toxicology 2004;42:455-6.

University of Versaille. Hyperbaric Oxygen Therapy for Acute Domestic Carbon Monoxide (CO) Poisoning. clinicaltrials.gov April 8 2010. [NCT01100515]

\section{Mathieu 1996 \{published and unpublished data\}}

Mathieu D, Wattel F, Mathieu-Nolf M, Durak C, Tempe JP, Bouachour G, Sainty JM. Randomized prospective study comparing the effect of $\mathrm{HBO}$ vs. 12 hours NBO in non-comatose CO-poisoned patients: results of the preliminary analysis. Undersea \& Hyperbaric Medicine 1996;23 Suppl:7 (abstract).

Raphael 1989 \{published and unpublished data\}

Raphael JC, Elkharrat D, Jars-Guincestre M-C, Chastang C, Chasles V, Vercken J-B, Gajdos P. Trial of normobaric and hyperbaric oxygen for acute carbon monoxide intoxication. Lancet 1989;2:414-9. [MEDLINE: 1989343396]

\section{Scheinkestel 1999 \{published and unpublished data\}}

* Scheinkestel CD, Bailey M, Myles PS, Jones K, Cooper JD, Millar IL, Tuxen DV. Hyperbaric or normobaric oxygen for acute carbon monoxide poisoning: a randomised controlled clinical trial. Medical Journal of Australia 1999;170:203-10. [MEDLINE: 1999192876]

Scheinkestel CD, Jones K, Copper DJ, Millar I, Tuxen DV, Myles PS. Interim analysis - controlled clinical trial of hyperbaric oxygen in acute carbon monoxide (CO) poisoning. Undersea \& Hyperbaric Medicine 1996;23(suppl):7.

\section{Thom 1995 \{published and unpublished data\}}

* Thom SR, Taber RL, Mendiguren II, Clark JM, Hardy KR, Fisher AB. Delayed neurologic sequelae after carbon monoxide poisoning: prevention by treatment with hyperbaric oxygen. Annals of Emergency Medicine 1995;25:474-80. [MEDLINE: 1995225522]

Thom SR, Taber RL, Mendiguren IL, Clark JM, Fisher AB. Delayed neurological sequelae following $\mathrm{CO}$ poisoning and the role of treatment with $100 \% 02$ or hyperbaric oxygen - A prospective randomized, clinical study. Undersea Biomedical Research 1992;19 (Suppl):47.
Thoms SR. Hyperbaric oxygen therapy for carbon monoxide poisoning: is it time to end the debates?. Toxicological Reviews 2005;24(3):157-8.

Weaver 2002 \{published data only\}

* Weaver LK, Hopkins RO, Chan KJ, Churchill S, Elliott CG, Clemmer TP, Orme JF, Thomas FO, Morris AH. Hyperbaric oxygen for acute carbon monoxide poisoning. New England Journal of Medicine 2002;347(14):1057-67.

Weaver LK, Hopkins RO, Larson Lohr V, Howe S, Haberstock D. Double blind, controlled, prospective, randomized clinical trial (RCT) in patients with acute carbon monoxide (CO) poisoning: outcome of patients treated with normobaric oxygen or hyperbaric oxygen (HBO) - an interim report. Undersea and Hyperbaric Medicine 1995;22(suppl):14.

Weaver LK, Hopkins RO, Valentine KJ, Churchill S. Persistent and delayed carbon monoxide cognitive sequelae. UHMS Meeting Abstracts 2005; http://archive.rubiconfoundation.org/1788.

Weaver LK, Valentine KJ, Hopkins RO. Carbon monoxide poisoning: risk factors for cognitive sequelae and the role of hyperbaric oxygen. American Journal of Respiratory and Critical Care Medicine 2007;176(5):491-7.

\section{References to studies excluded from this review}

Ducasse 1995 \{published and unpublished data\}

Ducasse JL, Celsis P, Marc-Vergnes JP. Non-comatose patients with acute carbon monoxide poisoning: hyperbaric or normobaric oxygenation?. Undersea and Hyperbaric Medicine 1995;22:9-15. [MEDLINE: 1995261254]

Gao 2002 \{published data only\}

Gao CJ, Ge H, Zhao LM, Wu LH, Xia CQ, Li Z, et al. Combined therapy with hyperbaric oxygen and anti-platelet aggregation agent for the prevention of delayed encephalopathy after acute carbon monoxide poisoning. Chinese Journal of Nautical Medicine and hyperbaric medicine 2002;9(3):142-4.

\section{Hampson 2006 \{published data only\}}

Hampson NB, Dunford RG, Ross DE, Wreford-Brown CE. A prospective, randomized clinical trial comparing two hyperbaric treatment protocols for carbon monoxide poisoning. Undersea \& Hyperbaric Medicine: Journal of the Undersea and Hyperbaric Medical Society, Inc. 2006;33(1):27-32.

\section{Hopkins 2007 \{published data only\}}

Hopkins RO, Weaver LK, Valentine KJ, Mower C, Churchill S, Carlquist J. Apolipoprotein E genotype and response of carbon monoxide poisoning to hyperbaric oxygen treatment. American journal of respiratory and critical care medicine 2007;176(10):1001-6. 
Weaver 2007 \{published data only\}

Weaver LK, Churchill S. One vs. Three Hyperbaric Oxygen Treatments for Acute Carbon Monoxide Poisoning. clinicaltrials.gov April 23, 2007.

\section{Additional references}

\section{Bassler 2010}

Bassler D, Briel M, Montori VM, Lane M, Glasziou P, Zhou Q, et al. Stopping randomized trials early for benefit and estimation of treatment effects: systematic review and meta-regression analysis. JAMA. 2010;303(12):1180-7.

\section{Buckley 2005a}

Buckley NA, Isbister GK, Juurlink DN. Hyperbaric Oxygen for Carbon Monoxide Poisoning: Evidence versus Opinion. Toxicological Reviews 2005;24(3):159-60.

\section{Bunc 2006}

Bunc M, Luzar B, Finderle Z, Suput D, Brvar M. Immediate oxygen therapy prevents brain cell injury in carbon monoxide poisoned rats without loss of consciousness. Toxicology 2006;225(2-3):138-41. [PUBMED: 16814444]

\section{Gilmer 2002}

Gilmer B, Kilkenny J, Tomaszewski C, Watts JA. Hyperbaric oxygen does not prevent neurologic sequelae after carbon monoxide poisoning. Academic emergency medicine: official journal of the Society for Academic Emergency Medicine 2002;9(1):1-8. [PUBMED: 11772662]

\section{Hampson 2004}

Hampson NB, Mathieu D, Thom SR, Weaver LK. Carbon monoxide poisoning: interpretation of randomized clinical trials and unresolved treatment issues. Undersea \& Hyperbaric Medicine 2001;28:157-64.

\section{Hampson 2007}

Hampson NB, Weaver LK. Carbon monoxide poisoning: a new incidence for an old disease. Undersea \& Hyperbaric Medicine: Journal of the Undersea and Hyperbaric Medical Society, Inc 2007;34(3):163-8.

\section{Hampson 2008}

Hampson NB, Hauff NM. Carboxyhemoglobin levels in carbon monoxide poisoning: do they correlate with the clinical picture?. The American journal of emergency medicine 2008;26(6):665-9. [PUBMED: 18606318]

\section{Higgins 2008}

Julian PT Higgins and Douglas G Altman on behalf of the Cochrane Statistical Methods Group and the Cochrane Bias Methods Group. Chapter 8: Assessing risk of bias in included studies. Cochrane Handbook for Systematic Reviews of Interventions. Version 5.0.0 [updated February 2008]. The Cochrane Collaboration, Available from www.cochranehandbook.org. The Cochrane Collaboration, 2008.

\section{Jay 1997}

Jay GD, McKindley DS. Alterations in pharmacokinetics of carboxyhaemoglobin produced by oxygen under pressure. Undersea \& Hyperbaric Medicine 1997;24:165-74.

\section{Logue 2008}

Logue CJ. An inconvenient truth?. Annals of emergency medicine 2008; Vol. 51, issue 3:339-40; author reply 340-2. [PUBMED: 18282535]

\section{Morrison 2010}

Morrison LJ, Deakin CD, Morley PT, Callaway CW, Kerber RE, Kronick SL, et al. Part 8: advanced life support: 2010 International Consensus on Cardiopulmonary Resuscitation and Emergency Cardiovascular Care Science With Treatment Recommendations. Circulation 2010;122(16 Suppl 2):S345-421. [PUBMED: 20956256]

\section{Raphael 2004}

Raphael JC, Chevret S, Driheme A, Annane D. Managing carbon monoxide poisoning with hyperbaric oxygen (Abstract). Journal of Toxicology - Clinical Toxicology 2004;42:455-6.

\section{Riley 2011}

Riley RD, Higgins JP, Deeks JJ. Interpretation of random effects meta-analyses. BMJ (Clinical research ed.) 2011;342:d549. [PUBMED: 21310794]

\section{Sackett 2001}

Sackett DL. Why randomized controlled trials fail but needn't: 2. Failure to employ physiological statistics, or the only formula a clinician-trialist is ever likely to need (or understand!). CMAJ : Canadian Medical Association journal = journal de l'Association medicale canadienne 2001;165(9):1226-37. [PUBMED: 11706914]

\section{Sanders 2009}

Sanders RW, Katz KD, Suyama J, Akhtar J, O'Toole KS, Corll D, et al. Seizure during Hyperbaric Oxygen Therapy for Carbon Monoxide Toxicity: A Case Series and Five-year Experience. Journal of Emergency Medicine 2009;ePub (in press):doi:10.1016/j.jemermed.2008.12.017.

\section{Stoller 2007}

Stoller KP. Hyperbaric oxygen and carbon monoxide poisoning: a critical review. Neurological Research 2007;29(2):146-55.

\section{Thom 2002}

Thom SR. Carbon monoxide-induced deficits in cognitive performance of mice and lack of effect of hyperbaric oxygen treatment. Academic Emergency Medicine 2002;9:75-7.

\section{Thom 2006}

Thom SR, Bhopale VM, Fisher D. Hyperbaric oxygen reduces delayed immune-mediated neuropathology in experimental carbon monoxide toxicity. Toxicology and applied pharmacology 2006;213(2):152-9. [PUBMED: 16325878]

\section{Vanden Hoek 2010}

Vanden Hoek TL, Morrison LJ, Shuster M, Donnino M, Sinz E, Lavonas EJ, et al. Part 12: cardiac arrest in special situations: 2010 American Heart Association Guidelines for 
Cardiopulmonary Resuscitation and Emergency Cardiovascular Care. Circulation 2010;122(18 Suppl 3):S829-61. [PUBMED: 20956228]

\section{Weaver 1995}

Weaver LK, Hopkins RO, Larson-Lohr A. Carbon monoxide poisoning: a review of human outcome studies comparing normobaric oxygen with hyperbaric oxygen. Annals of Emergency Medicine 1995;25(2):271-2.

\section{Weaver 1995a}

Weaver LK, Hopkins RO, Larson-Lohr V, Howe S, Haberstock D. Double blind, controlled, prospective, randomized clinical trial (RCT) in patients with acute carbon monoxide (CO) poisoning: Outcome of patients treated with normobaric oxygen or hyperbaric oxygen (HBO2) - An interim report. Undersea \& Hyperbaric Medicine. 1995; Vol. 22 (Suppl):14.

\section{Weaver 1999}

Weaver LK. Carbon monoxide poisoning. Critical Care Clinics 1999;15:297-317.

\section{Weaver 2001}

Weaver LK, Churchill S. Pulmonary edema associated with hyperbaric oxygen therapy. Chest. 2001;120(4):1407-9.

\section{Weaver 2005}

Weaver LK, Hopkins RO, Valentine KJ, Churchill S. Persistent and delayed carbon monoxide cognitive sequelae. UHMS Meeting Abstracts. 2005; Vol. http://archive.rubiconfoundation.org/1788.

\section{CHARACTERISTICS OF STUDIES}

Characteristics of included studies [ordered by study ID]

\section{Weaver 2007}

Weaver LK, Valentine KJ, Hopkins RO. Carbon monoxide poisoning: risk factors for cognitive sequelae and the role of hyperbaric oxygen. American journal of respiratory and critical care medicine 2007;176(5):491-7. [PUBMED: 17496229]

\section{Weaver 2009}

Weaver LK. Clinical practice. Carbon monoxide poisoning. New England Journal of Medicine 2009;360(12):1217-25.

\section{Wolf 2008}

Wolf SJ, Lavonas EJ, Sloan EP, Jagoda AS. Clinical policy: Critical issues in the management of adult patients presenting to the emergency department with acute carbon monoxide poisoning. Annals of Emergency Medicine 2008;51(2):138-52.

\section{References to other published versions of this review \\ Buckley 2005 \\ Buckley NA, Isbister GK, Stokes B, Juurlink DN. Hyperbaric oxygen for carbon monoxide poisoning: a systematic review and critical analysis of the evidence. Toxicological Reviews 2005;24(2):75-92.}

\section{Juurlink 2005}

Juurlink D, Buckley N, Stanbrook M, Isbister G, Bennett M, McGuigan M. Hyperbaric oxygen for carbon monoxide poisoning. The Cochrane Database of Systematic Reviews 2005, Issue 1. [DOI: 10.1002/14651858.CD002041.pub2]

* Indicates the major publication for the study

\section{Annane 2010}

Methods

Prospective, randomised unblinded trial. Similar to the earlier trial by the same investigators, randomisation was stratified by history of "transient loss of consciousness" vs. "initial coma". Patients without impaired consciousness were excluded. Patients with "transient loss of consciousness" were randomised to HBO vs NBO ("Trial A") and are included in this review. A separate group of patients with "initial coma" was randomised to receive 1 vs. 2 HBO treatment sessions ("Trial B"), and are not considered in this review.

The terms "transient loss of consciousness" and "initial coma" were defined by the authors as follows: "Eligible patients were classified by [the] attending physician into those who experienced transient loss of consciousness (malaise, syncope; trial A) or coma as confirmed by a household member or a rescuer (trial B)." "Coma was defined as a Glasgow coma score of $<8$. Transient loss of consciousness or malaise or syncope was defined as normal consciousness at the time the patient was rescued and he/she could not recall what happened and/or reported that he/she had loss [of] consciousness." However, the definitions were difficult to apply; 14 people with initial impaired consciousness were unable to be classified into either group and were excluded from both studies, 6 patients randomised in Trial A should have been randomised in Trial $B$, and 12 patients randomised in Trial $B$ should have been randomised in Trial A.

Participants

179 patients $\geq 15$ years of age presenting for therapy between Oct 1989 and Jan 2000 within 12 hours of exposure with a $\mathrm{COHb}$ of $>5 \%$ if a non-smoker or $>10 \%$ if a smoker and a history of transient (but not 
Annane 2010 (Continued)

sustained) loss of consciousness. Key exclusion criteria included: suicide attempt, non-domestic poisoning, inhalation of smoke or other toxic gasses other than $\mathrm{CO}$, and pregnancy.

Interventions

In "Trial A", patients with "transient loss of consciousness" were randomised to receive mask oxygen alone for 6 hours (NBO) or mask oxygen for 4 hours and HBO at 2.0 ATA for 120 minutes including 30 minutes compression/decompression. In addition, $\mathrm{HBO}$ patients received diazepam $10 \mathrm{mg}$ IM.

Outcomes

The primary study endpoint was the proportion of patients with complete recovery 1 month after poisoning. Quote: "Complete recovery was defined as an absence of symptoms reported on the self-assessment questionnaire with a normal physical exam (including normal neuropsychological functions). These assessments were performed "by one intensive care physician qualified in neurology who remained blinded to patients' treatment arm," or by the patient's primary care physician. The type of neuropsychological testing employed and thresholds considered abnormal are not described. Sequelae were defined as "moderate" if they consisted of patient-reported symptoms only, and "severe" if any abnormalities were detected on examination. Key secondary outcomes included the proportion of patients with persistent or delayed neurological sequelae and the proportion able to resume their former occupational activity.

No difference in the primary or key secondary outcomes were evident, with symptoms present in 33/79 (42\%) HBO patients 29/74 (39\%) of control patients one month after poisoning/treatment. These results remained statistically insignificant, with the point estimate slightly favouring NBO, following adjustment for sex, duration of intoxication, time to randomisation and initial $\mathrm{COHb}$ level.

Notes This trial was originally reported in abstract in 2004 (Raphael 2004) and included in our previous review. The trial protocol was retrospectively added to a clinical trials registry (https://register.clinicaltrials.gov with number NCT01100515 for trial A). Some additional unpublished information was made available from a 1989 protocol provided by the authors. Trial stopped because interim analysis of Trial $B$ indicated $2 \times \mathrm{HBO}$ treatments were inferior to one ( $47 \%$ with complete recovery vs $68 \%$ respectively). Trial A stopped at the same time as interim analysis indicated the results were close and suggested "futility of treatment continuation".

Although "trial B" of this study did not meet inclusion criteria for this review, it can be used to examine whether a dose/duration-response effect of HBO is apparent. In "trial B" (a more seriously poisoned group than the above), the proportion of patients with residual symptoms 1 month after poisoning was greater among patients who received two HBO sessions (53\%) than in those treated with one HBO session (32\%), suggesting harm associated with an increased total duration of $\mathrm{HBO}$.

\section{Risk of bias}

\begin{tabular}{lll}
\hline Bias & Authors' judgement & Support for judgement \\
\hline $\begin{array}{l}\text { Adequate sequence gener- } \\
\text { ation? }\end{array}$ & Low risk & $\begin{array}{l}\text { Quote: "An independent statistician prepared a computer-generated alloca- } \\
\text { tion sequence for each trial (A and B)." }\end{array}$ \\
\hline Allocation concealment? & Low risk & $\begin{array}{l}\text { Quote: "Patients were randomised by using numbered sealed envelopes." } \\
\text { Comment: The authors do not report if there was cluster randomisation or } \\
\text { how groups of patients who presented simultaneously from the same incident } \\
\text { were randomised. The authors do not report if blocking was used [although } \\
\text { this seems unlikely as there was an imbalance (86 vs 93)]. }\end{array}$ \\
\hline
\end{tabular}

Blinding? High risk

All outcomes

Quote: "At 1 month, patients completed a self-assessment questionnaire with yes/no items about headaches, tiredness, memory impairment, difficulty in concentrating, difficulty in sleeping, visual disorders, and new difficulties with social or professional activities. They underwent a thorough physical examination at the ICU outpatient clinic by one intensive care physician qualified in neurology who remained blinded to patients' treatment arm."

Comment: The patient and treating team were not blinded as there was no attempt at sham treatment. While physical examination was said to be blinded 


\section{Incomplete outcome data Low risk} addressed?

All outcomes
Quote: "At 1 month, 26 patients (14.5\%) were lost to follow-up."; "sensitivity analyses yielded consistent findings when lost to follow-up patients were classified as failures $[\mathrm{OR}=0.89(\mathrm{Cl}, 0.50-1.60)]$ or as successes $[\mathrm{OR}=0.93(\mathrm{Cl}, 0.50$ 1.71)], and regardless of the method used to treat missing values (Table 3 )."
Free of selective report- Unclear risk ing?
The trial was retrospectively registered after completion. The results are consistent with an abstract presented in 2004. All important clinical secondary outcomes were presented. However the original 1989 protocol (in French) suggested some other outcomes were to be recorded [paraphrased from translation of the unpublished protocol] "Assessment of outcomes would include....the fate of patients (back at home, convalescent and nursing homes), duration of hospitalisation, changes in muscle enzymes and liver function, chest x-ray changes, electrocardiogram changes including secondary manifestations (arrhythmias, conduction, appearance of disorders of repolarization, abnormal QRS complex) and electroencephalogram changes (possible outcome).

Comment: None of these outcomes were likely to be common in Trial A as the overall outcome was very good (only $1 / 179$ patients not resuming former activity). The outcomes of interest to this review were reported in full.
Free of other bias? Unclear risk Power/Sample size/stopping rules:

Quote: "Sample sizes for trial A and B were calculated separately. In trial A, assuming a 1 month recovery rate of $40 \%$ in the reference group (A0), 245 patients were needed per treatment arm to detect an absolute difference of at least $15 \%$ in recovery rates with a type I error of 0.05 and a type II error of 0.10 using a two-sided test."

Quote: "One interim analysis was scheduled after inclusion of 300 patients to allow premature study termination if data suggested a large difference between treatment arms, serious treatment-related adverse events, difficulties in conducting the trial, or futility".

Quote: "The trial was terminated prematurely after the interim analysis (January 2000), based on a total of 385 patients, showing that, in comatose patients (trial B), the 1 month complete recovery rate was lower in the "two HBO sessions" arm compared to the "one HBO session" arm (47 vs. $68 \%, P=0.007)$. Furthermore, in trial A patients, recovery rates were close (61\% in control arm vs. $58 \%$ in experimental arm), suggesting futility of treatment continuation. Accordingly, we decided to terminate trial A also."

Comment: Trial A appears to have been stopped largely on the basis of the results from Trial B which was poorly justified. However, while this reduced the power of the study, it is unlikely to lead to a bias.

\section{Mathieu 1996}

\begin{tabular}{ll}
\hline Methods & Prospective, randomised, unblinded trial. \\
\hline Participants & $\begin{array}{l}575 \text { non-comatose nonpregnant patients with no evidence of mixed poisoning, recruited over } 3 \text { years. } \\
\mathrm{COHb}>10 \% .\end{array}$ \\
\hline Interventions & $\begin{array}{l}\text { HBO at } 2.5 \text { ATA for } 90 \text { minutes (plus } 15 \text { minutes each for compression and decompression) vs. } 12 \text { hours } \\
\text { of NBO. }\end{array}$ \\
\hline
\end{tabular}


Mathieu 1996 (Continued)

Outcomes

Neuropsychologic testing at 1, 3, 6, and 12 months. 'Persistent neurological manifestations' were present in $23 \%$ of $\mathrm{HBO}$ arm and $26 \%$ of NBO arm at 1 month, but detailed data were not presented.

Notes

Data from abstract of 1996 interim analysis only. This trial is not registered and no later data were available for analysis at the time of the 2005 or 2011 review. Author contacted in 2004 and 2010 but no further information provided.

\section{Risk of bias}

\begin{tabular}{lll}
\hline Bias & Authors' judgement & Support for judgement \\
\hline $\begin{array}{l}\text { Adequate sequence gener- } \\
\text { ation? }\end{array}$ & Unclear risk & $\begin{array}{l}\text { Quote: "After randomisation patients are treated either by a HBO session (2.5 } \\
\text { ATA, } 90 \text { min) or by } 12 \text { hours normobaric pure oxygen." }\end{array}$ \\
\hline Allocation concealment? & Unclear risk & Not mentioned. \\
\hline $\begin{array}{l}\text { Blinding? } \\
\text { All outcomes }\end{array}$ & High risk & Not mentioned. Without sham therapy blinding would not be possible. \\
\hline
\end{tabular}

\section{Incomplete outcome data High risk} addressed?

All outcomes
Quote: "At 1 month, follow-up shows fewer (although not statistically significant) persistent neurologic manifestations in the HBO group than in the NBO (23\% vs 26\%). At 3 months, a large significant difference exists (HBO: $9.5 \%$ vs NBO: $15 \% ; p=0.016)$. The difference becomes less important at 6 months $(6.4 \%$ vs $9.5 \%$; $p=0.09)$ and disappears at 1 year (4.3\% vs $5 \%)$."

Comment: No information on drop-outs provided.
Free of selective report- Unclear risk ing?

\begin{abstract}
Quote: "Patients were all discharged from the hospital with a normal neurologic examination. At 1 month, follow-up shows fewer (although not statistically significant) persistent neurologic manifestations in the HBO group than in the NBO (23\% vs $26 \%)$. At 3 months, a large significant difference exists (HBO: $9.5 \%$ vs NBO: $15 \% ; p=0.016$ ). The difference becomes less important at 6 months $(6.4 \%$ vs $9.5 \% ; p=0.09)$ v and disappears at 1 year ( $4.3 \%$ vs $5 \%) . "$

Quote: "The study is continued to allow proper subgroup analysis."

Comment: Abstract published in 1996, the final report or a later interim analysis has not been presented in any forum.
\end{abstract}

Power/sample size/interim analysis/publication:

Quote: "An interim analysis was planned at the end of the third year".

"The study is continued to allow proper subgroup analysis".

Comment: No sample size calculation. No response to request for further information in 2004 or 2010.

\section{Raphael 1989}

Methods

Prospective, randomised, unblinded trial. Randomization stratified according to history of loss of consciousness (LOC). Allocation by sealed opaque envelopes, not sequentially numbered. Only those with no history of $\mathrm{LOC}$ randomised to $\mathrm{HBO}$ vs. NBO; more severe patients randomised to different regimens of $\mathrm{HBO}$. Only the $\mathrm{HBO}$ vs NBO group included in this review.

Participants

629 adults admitted within 12 hours of termination of $\mathrm{CO}$ exposure. Inclusion: age >15 years, admitted within 12 hours, $\mathrm{COHb}>10 \%$ (smoker) or $5 \%$ (nonsmoker). Exclusion: other intoxication, pregnancy, cardiovascular collapse, pulmonary edema, non-feasible HBO (technical problems etc.), difficulty in 
Raphael 1989 (Continued)

stratifying into groups A or B (by LOC), refusal by patient. Of enrolled patients, 343 were randomised to receive either $\mathrm{HBO}$ or $\mathrm{NBO}$.

Interventions

Only those without history of loss of consciousness randomised to HBO [HBO for $2 \mathrm{~h}$ followed by $100 \%$ oxygen by mask for $4 \mathrm{~h}$ (where $\mathrm{HBO}$ regimen included 30 minutes compression \& decompression flanking 60 minutes at 2.0 ATA.)] vs. NBO [ $100 \%$ oxygen by mask for $6 \mathrm{~h}]$. Other patients randomised to $\mathrm{HBO} x$ 1 vs. $\mathrm{HBO} \times 2$ not included in this review's analysis.

\begin{tabular}{|c|c|}
\hline Outcomes & $\begin{array}{l}\text { Intention to treat analysis. Outcome measures included self-assessment questionnaire and physi- } \\
\text { cal examination by neurologist (unblinded) at one month, with no difference in outcome (symptoms } \\
\text { present in } 50 \text { of } 158 \text { patients ( } 32 \%) \text { treated with NBO vs. } 51 \text { of } 159 \text { patients (32\%) treated with HBO at } \\
\text { one month). }\end{array}$ \\
\hline Notes & $\begin{array}{l}\text { The second part of this study involving patients who had initial impairment of consciousness did not } \\
\text { meet inclusion criteria for this review. In this group of patients, the proportion with residual symptoms } \\
1 \text { month after poisoning was greater among patients who received two HBO sessions ( } 48 \% \text {; group B2) } \\
\text { than in those treated with one HBO session ( } 46 \% \text {; group B1). This difference was not statistically signifi- } \\
\text { cant and the trend did not provide any support for a dose-response relationship for HBO. }\end{array}$ \\
\hline
\end{tabular}

\section{Risk of bias}

Bias Authors' judgement Support for judgement

Adequate sequence gener- Unclear risk ation?

\begin{tabular}{ll}
\hline Allocation concealment? & Unclear risk \\
\hline Blinding? & High risk \\
All outcomes &
\end{tabular}

Quote: "Treatment was randomly selected by means of sealed envelopes and randomisation was stratified according to patient group."

\section{No mention of concealment strategies.}

Quote (from discussion): "A double-blind trial with sham HBO therapy might have been the ideal solution but both feasibility and security problems would have made it impossible to conduct such a study on a large scale."

No record of attempts to blind participants or assessment. The descriptions of the outcome measures employed were vague and therefore potentially open to interpretation by the unblinded assessors.

Incomplete outcome data Low risk
addressed?

All outcomes

Outcome data reported on 317 of the 343 patients randomised.

Quote "The differences remained non significant even when all patients lost to follow-up were assumed to have recovered or when all were assumed to have been left with sequelae."

Free of selective report- Low risk
ing?
Intent to treat analysis:

Quote: "Patients were not informed of the randomisation procedure because informed consent would have been difficult to obtain in an emergency setting and was not required by French regulations at the time. However, the treatment to be given was explained in detail to the patient (or family if the patient was comatose). If patients refused the allocated treatment after randomisation they were still retained in the study and analysed according to the treatment intended"

Quote "Most patients received the treatment allocated. 9 patients ( $3 \mathrm{Al}, 2 \mathrm{Bl}, 4$ $\mathrm{B} 2$ ) refused $\mathrm{HBO}, 2$ group $\mathrm{AO}$ received one $\mathrm{HBO}$ session by mistake". 
Scheinkestel 1999

Methods

Prospective double-blind RCT of HBO vs. NBO. Cluster randomisation for patients presenting simultaneously. Allocation through sealed opaque envelopes, not sequentially numbered. Patients and outcome assessor blind to allocation, technicians and nurses not. Stratified by vent/non-vent and suicide vs. accidental exposure.

Participants

230 patients sequentially referred to single centre in Australia. Inclusion: all referred. Excluded ( $n=39$ ): children, burn victims, pregnant, refusal of consent. Two groups similar for all important variables. $89 \%$ male, coma in $50.6 \%$, average $\mathrm{COHb} 21 \%$. Large number of suicide attempts (69\%), co-intoxication (44\%), and severe poisonings (73\%).

Interventions

All patients given high-flow $\mathrm{O} 2$ prior to randomisation. Randomized to daily treatment (for 3 days) with HBO (100 minutes; 60 minutes at 2.8 ATA) vs. NBO (100 minutes of $100 \% 02$ at 1 ATA) as a sham dive. All patients received $100 \%$ oxygen via mask continuously between sessions (i.e. $100 \%$ oxygen for more than 2 days). After third treatment, patients with deficits were treated again, with high-flow oxygen in between. 3 additional courses of original therapy given to $28 \% \mathrm{HBO}$ and $15 \%$ NBO because of "poor outcome".

Outcomes

191 randomised (104 HBO and NBO 87, discrepancy due to cluster.) No mortality difference at discharge. Poor follow-up attendance (46\%) at one month. Objective outcome data at 1 month post-treatment were not presented but it was stated that they "showed no difference in any test". Communication with the authors clarified that $34 / 52$ were symptomatic in $\mathrm{HBO}$ arm vs. $20 / 34$ symptomatic in NBO arm at 4 weeks $(p=N S)$ and this is the outcome we include in this meta-analysis.

Notes

\section{Risk of bias}

\section{Bias Authors' judgement Support for judgement}

Adequate sequence gener- Low risk ation?
Quote: "Patients were randomly allocated to HBO or NBO treatment"

Quote: "Patients were first stratified into four groups (suicide versus accidental, then mechanically ventilated versus non-ventilated). A hyperbaric technician then allocated patients to treatment groups by opening envelopes chosen from random blocks, each with equal numbers of HBO and NBO selections."

Allocation concealment? Unclear risk

Quote: "We used cluster randomisation for patients who presented simultaneously from the same $\mathrm{CO}$ exposure, allocating them all to the same treatment group".

Comment: It is unclear whether the randomisation of the cluster took place after the first person was enrolled (which would have likely meant there was no allocation concealment for all others in the cluster) or whether the entire cluster was screened for eligibility and consented prior to randomisation of the entire cluster which would have preserved allocation concealment.

$\begin{array}{ll}\begin{array}{l}\text { Blinding? } \\ \text { All outcomes }\end{array} & \begin{array}{l}\text { Quote: "The hyperbaric technicians and nursing staff had knowledge of the } \\ \text { treatment group but patients and outcome assessor did not." }\end{array} \\ & \begin{array}{l}\text { Comment: } 8 \text { patients had complications apparently related to HBO. It is like- } \\ \text { ly this would have effectively unblinded the analysis. The authors do not dis- } \\ \text { cuss whether the patient or physician was also unblinded in these cases, or } \\ \text { whether the patients completed the assigned therapy. No attempt was de- } \\ \text { scribed to assess whether the sham therapy was successful in maintaining } \\ \text { blinding. }\end{array}\end{array}$

Incomplete outcome data Unclear risk

addressed?

All outcomes
Quote: "Only $46 \%$ of patients attended the one-month follow-up. Thus, the numbers in subgroups of interest at one month were small, but showed no difference in any test between HBO and NBO groups". 
"Patients with poor outcome at hospital discharge were considered to have persistent neurological sequelae (PNS). Delayed neurological sequelae (DNS) were defined as morbidity found at follow-up that was not obvious at hospital discharge, or deterioration of neuropsychological subtest scores by more than one standard deviation."

"Poor outcome (PNS) HBO: 0.74 (of 104) NBO: 0.68 (of 87), OR: 1.7 (0.8-4.0) $\mathrm{P}=0.19^{\prime \prime}$.

Comment: No sensitivity analysis performed. Exact numbers for which assumptions are made are unclear from the final report.

Free of selective report- High risk ing?
Quote: "The only statistically significant difference between groups in neuropsychological performance was in the learning test at completion of treatment (Boxes 3 and 4); this was in favour of the NBO group for both 'all patients' $(P=0.01)$ and 'severely poisoned' patients $(P=0.005) "$

Quote: "NBO patients had a significantly lower number of abnormal test results at completion of treatment (all patients, 3.4 v. 2.7, $p=0.02$; severely poisoned patients, 3.7 v. 2.6, $\mathrm{P}=0.008$ ) and, for those severely poisoned, there were fewer NBO patients with a poor outcome $(85 \% \mathrm{v} .65 \%$; $P=0.03)$. All five relapses (DNS) occurred in HBO patients $(P=0.03)$ at a median of 40 days (IQR, 29-81 days) after initial treatment; these patients then received a mean 4.5 (SD, 2.5) additional treatments. Although three of these patients improved with further treatments, all DNS patients had a poor outcome after re-treatment, with a mean 6.3 (SD, 1.2) abnormal test results."

Comments:

Several of these and other conclusions in text were based upon analysis of individual neuropsychological test results in one sub-group. However, there was no adjustment for multiple comparisons leading to a high likelihood of a Type l error.

There was no pre-specified primary outcome in the paper. The focus of most of the analysis on early outcomes (immediately post-treatment) contrasted with their stated aim to "to assess both persistent and delayed neurological sequelae (PNS and DNS)" and is likely to have been driven by necessity given the low follow up (and therefore post hoc).
Free of other bias?
Unclear risk
Intention to treat/Power/Premature stopping-

Quote: "The Alfred Hospital's Ethics Committee approved the trial, conditional on an independent blinded interim analysis after recruitment of 50 patients (using a stopping rule of $\mathrm{P}<0.001$ )"

Quote: "Based on the most sensitive neuropsychological test (Short reaction time), with 191 patients and a significance level of 0.05 , we had greater than $99 \%$ power to detect a $10 \%$ difference between groups (i.e., 408 seconds v. 450 seconds; SD, 63 seconds)"

Interim analysis abstract (introduction) Quote: "Ethics Committee required an interim analysis of this prospective single blind study (with outcome assessor also blind to group identity) after 100 patients, to ensure early detection of any adverse outcomes." (145 patients recruited at this analysis, No P value presented)

Interim analysis abstract (results) Quote: "For the same exposure, time to treatment, time to CO level, CO level, initial mini-mental, signs and symptoms, the NBO group required less treatments and had a better outcome" "Subanalysis of severe $\mathrm{CO}$ poisoning ( $56 \mathrm{HBO}, 50 \mathrm{NBO}$ ) showed HBO group had more severe exposure and worse outcome" 
Interim analysis abstract (conclusions) Quote:"Interim analysis by major categories and sub-categories showed no difference in outcome for NBO and HBO treated groups."

Comment: The power calculation appears to be retrospective so it is unclear whether the trial was completed as planned or stopped prematurely, based on these results of the interim analysis.

\section{Thom 1995}

$\begin{array}{ll}\text { Methods } & \text { Prospective, randomised, unblinded trial of HBO vs. NBO. Treatment allocation by computer-generat- } \\ \text { ed random numbers within sealed opaque envelopes, not sequentially numbered. }\end{array}$
ed random numbers within sealed opaque envelopes, not sequentially numbered.

Participants patients referred from local emergency departments, within 6 hours of removal from exposure. In-
clusion criteria: history of acute exposure, elevated COHb, symptoms consistent with CO poisoning. Ex-
clusion criteria: history of loss of consciousness, active ischaemia. The two groups were similar with the
main clinical features being headache, nausea and lethargy and a mean COHb 20 to $25 \%$.

Interventions All patients in HBO arm given 100\% 02 until HBO initiated. HBO begun within 6 hours of end of exposure. HBO @ 2.8 ATA for 30 minutes, then 2.0 ATA x 90 minutes. NBO 100\% O2 until all symptoms resolved (mean $4.2+/-0.3$ hours). After intervention, neuropsychologic baseline testing ( 6 tests) performed (some up to 12 hours post-treatment).

$\begin{array}{ll}\text { Outcomes } & \text { Occurrence of DNS self-reported as (1) recurrent symptoms or (2) new symptom consistent with DNS, } \\ \text { plus deterioration in } 1 \text { or more subtest upon retesting. Outcome assessors not blind to treatment allo- } \\ \text { cation. } 5 \text { patients lost to follow up ( } 2 \text { control, } 3 \text { HBO). } 7 / 30 \text { patients in control arm had sequelae consis- } \\ \text { tent with DNS vs. 0/30 patients in HBO arm. }\end{array}$

Notes

\section{Risk of bias}

Bias Authors' judgement Support for judgement

Adequate sequence gener- Unclear risk Quote: "Patients were randomly assigned to one of two treatment arms"
ation?
ation?

\begin{tabular}{|c|c|c|}
\hline Allocation concealment? & Unclear risk & Not mentioned \\
\hline $\begin{array}{l}\text { Blinding? } \\
\text { All outcomes }\end{array}$ & High risk & Quote: "Neither the patients nor investigators were blinded to treatment:" \\
\hline
\end{tabular}

\begin{tabular}{|c|c|c|}
\hline $\begin{array}{l}\text { Incomplete outcome data } \\
\text { addressed? } \\
\text { All outcomes }\end{array}$ & Low risk & $\begin{array}{l}\text { Quote: "Two patients in the ambient-pressure group and three in the HBO } \\
\text { group were lost to follow-up. Two patients in each treatment group refused } \\
\text { formal neuropsychologic retesting but denied symptoms of DNS during tele- } \\
\text { phone interviews conducted over the next } 3 \text { months." }\end{array}$ \\
\hline
\end{tabular}

Comment: While the incomplete outcomes were reported, no sensitivity analysis was performed by the authors as to whether this might have influenced the findings of this trial.

$\begin{array}{ll}\begin{array}{ll}\text { Free of selective report- } & \text { Unclear risk }\end{array} & \text { Quote: "Deteriorations in the DNS group scores occurred in three sub-tests: } \\ \text { ing? } & \text { Trail Making, Digit Symbol, and Block Design. The mean changes in scores } \\ & \text { were significantly different from those of a matched control group (Figure } \\ & \begin{array}{l}\text { 1). No differences were found in sub-tests on General Orientation, Digit Span } \\ \text { recitals, or Aphasia Screening (results not shown)." }\end{array}\end{array}$


Thom 1995 (Continued)

Quote: "In eight patients in the ambient-pressure group, symptoms consistent with DNS developed. Seven of these patients also had deterioration in at least one subtest category and were considered to have DNS".

Comment: There was no pre-specified primary outcome in the paper.

Free of other bias? High risk

\author{
Intention to treat/Power/Premature stopping- \\ Quotes (from 1992 abstract): "This is a preliminary report of an on-going \\ study".
}

"To date, 27 patients have been entered, and 2 lost to follow-up, in each treatment arm".

"Following hyperbaric 02 treatment, no patients have suffered deteriorations ( $p<0.005, x^{2}$ - two-tail test)."

Quote from 2005 letter: "We did publish a preliminary report on our trial in 1992. At the time of that report, 27 patients had been randomised. Four patients treated with normobaric oxygen (the control group) had neurological sequelae, whereas none among those treated with HBO had sequelae".

Comment: No power/sample size calculation or stopping rule was specified in the paper. There is no feasible power calculation that would have planned a study this size. [Also a P value of $<0.005$ simply cannot be generated from a chi square test with 4 outcomes in 27 patients (see Buckley 2005a for further exposition on this point)]. It seems likely the trial was stopped early based upon ongoing serial analysis of the available data. There was no statistical adjustment for multiple comparisons, indicating a high probability of Type I error.

In any case, premature stopping of small trials "for benefit" inevitably greatly exaggerates the observed effect for statistical reasons (Bassler 2010).

Weaver 2002

Methods $\quad$ Prospective, randomised, double-blind RCT of HBO vs. NBO.

Participants

152 patients with $\mathrm{CO}$ poisoning (symptomatic and $\mathrm{COHb}>10 \%$ or symptoms and signs unequivocally due to $\mathrm{CO}$ exposure). Exclusions: Pregnancy, $>24 \mathrm{~h}$ since exposure, $<16$ years of age, moribund, refused consent. Stratified by level of consciousness, age $<40$, and delay to treatment $<6 \mathrm{~h}$.

Patients were primarily exposed to CO from internal combustion engines, furnaces, and heaters. $31 \%$ of cases involved suicide attempts. A subsequently-published analysis showed that patients who were eligible for this trial but were not enrolled had similar demographics, exposure characteristics, and outcomes to the NBO patients in the trial (Weaver 2007).

Patients enrolled in the NBO arm appeared more ill than those in the HBO arm, with a longer mean exposure ( 22 hours vs. 13 hours) and a greater prevalence of cerebellar signs at baseline ( $15 \%$ vs. $4 \%$, respectively). Cerebellar problems would interfere with performance in the two trail-making tests [the authors reported an adjusted analysis that included baseline cerebellar signs as a covariate during the final statistical analysis].

Interventions HBO - 1 session 3ATA $x$ 1h \& 2ATA $x$ 1h, followed by two sessions 2ATA $x$ 2h at $6-12$ hour intervals. NBO patients received sham treatment at 1 ATA. Oxygen not routinely used after first session.

Outcomes

Structured questionnaire, serial neurological testing, and other formalized assessments (e.g. Geriatric Depression Scale, Katz index of activities of daily living, SF-36) immediately after treatments 1 and 3, and then at 2 and 6 weeks follow-up. 
Data from 26 and 52 weeks treatment was obtained from a different long-term follow-up study that included a majority of these patients.

\section{Risk of bias}

\begin{tabular}{lll}
\hline Bias & Authors' judgement & Support for judgement \\
\hline $\begin{array}{l}\text { Adequate sequence gener- } \\
\text { ation? }\end{array}$ & Low risk & $\begin{array}{l}\text { Quote: "Patients were randomly assigned to receive hyperbaric-oxygen thera- } \\
\text { py or normobaric-oxygen therapy with the use of blocked, stratified randomi- } \\
\text { sation with allocation determined by a list of computer-generated random } \\
\text { numbers"; "The block size was six, and patients were stratified........" }\end{array}$ \\
& & $\begin{array}{l}\text { Comment: Allocation concealment could feasibly be jeopardized by using a } \\
\text { fixed block size (if this was known). }\end{array}$ \\
\hline Allocation concealment? & Low risk & $\begin{array}{l}\text { Quote: "Treatment-group assignments were given to respiratory therapists in } \\
\text { protected, sequentially numbered, sealed, opaque envelopes". }\end{array}$ \\
\hline
\end{tabular}

Blinding? Low risk

All outcomes

\section{Blinding of patients and outcome assessment:}

Quote: "Patients in the normobaric-oxygen group were exposed to air at 1 atmosphere absolute (101.3 kPa, or sea-level pressure) for all three chamber sessions. The chamber was pressurized to sea-level pressure in order to maintain blinding of patients and investigators"; "Respiratory therapists operated the controls for the chamber while observing pressure gauges visible only to them. These therapists maintained separate confidential records of the chamber sessions in order to ensure that others were unaware of the treatment group assignments".

Comment: Trial conducted in Utah, thus pressurising to sea level led to sensation of pressure in sham treatment (NBO) group. Respiratory therapists not blinded but not involved in other aspects of trial conduct.

Blinded analysis:

"All data were analysed by investigators and statisticians who were blinded to the treatment-group assignments."

"The statisticians and investigators, who were blinded to patients' treatment-group assignments, had access to the results of the interim analyses."

"Failure to complete the chamber sessions was more common in the hyperbaric-oxygen group (14 of 76 [18.4 percent]) than in the normobaric-oxygen group ( 3 of 76 [3.9 percent], $\mathrm{P}=0.005$ ). The first hyperbaric-oxygen treatment was stopped prematurely because of anxiety (in seven patients), tympanic-membrane rupture (in one patient), and cough (in one patient). The second or third hyperbaric-oxygen treatment was omitted due to difficulty with equalization of middle-ear pressure (in four patients) or failure to return for treatment (in one patient). The second or third normobaric-oxygen session was omitted because of failure to return (in three patients)."

Comment: Chamber related adverse events expected to be much more common with $\mathrm{HBO}$ than sham treatment. This may have meant which patients were in the $\mathrm{HBO}$ arm was apparent during subsequent analysis of efficacy data.

Incomplete outcome data Low risk

4 - 6 week time point

addressed?

All outcomes
Quote: "The five patients who did not have data on neuropsychological tests at six weeks were assumed to have cognitive sequelae at that time point." 
Comment: This assignment of an adverse effect to those with incomplete data applied to 4 NBO patients and 1 HBO patient. This introduced a bias in favour of a beneficial effect of $\mathrm{HBO}$. No sensitivity analysis was done to test whether the conclusions were sensitive to this assumption. This also has impact on interim analysis stopping rules given the $p$ value for stopping was 0.01 and this level of significance was only just reached (i.e. the trial would not have stopped if this particular assumption had not been applied).

6 and 12 month time points:

Quotes from 2002 abstract:

"Although this RCT did not measure 6 and 12 month outcome, we had data from most of these RCT patients from a separate IRB-approved study permitting us to report outcome at 6 and 12 months post-CO poisoning."

"The analysis assumed a "favorable outcome" if: 1) No cognitive sequelae at all times ( $\mathrm{n}=29-\mathrm{HB} 02 / / 22-\mathrm{NB} 02) ; 2$ ) 6-week cognitive sequelae present, but resolved by 12 months $(n=5 / / 9) ; 3$ ) No cognitive sequelae at 6 weeks, and either no data at 6 or 12 months, and no cognitive sequelae present ( $n=13 / / 8)$; or 4 ) Cognitive sequelae developing after 6 weeks was not due to the prior episode of CO poisoning $(n=15 / / 11)$."

Comment: This abstract clarifies that those with documented normal results at the 12 month time point were 34 for HBO vs 31 for NBO and by adding the 4 th group to the numbers reported in the NEJM paper those with documented abnormal results were 24 for HBO vs 29 for NBO (Neither of these results would be significant).

\section{Quotes from NEJM paper:}

"We also report rates of cognitive sequelae at 6 and 12 months, although these end points were not included in the study design."

"Cognitive sequelae present at 6 or 12 months were assumed not to be due to carbon monoxide poisoning if they had not been present at 6 weeks. Patients with cognitive sequelae at 6 weeks who had missing data at 6 or 12 months were assumed to have cognitive sequelae at those time points."

Comment: These two assumptions meant that any differences at the first time point were likely to be carried forward to all later analyses. So not only those who did not attend the $6(n=35)$ and $12(n=24)$ month assessments had the 6 week assessment carried forward, but so did those who had normal results at 6 weeks (57/76 (HBO) vs. 41/76 (NBO)).

"Cognitive sequelae at 6 months and 12 months were less frequent in the hyperbaric-oxygen group than in the normobaric-oxygen group, both according to the intention-to-treat analysis $(P=0.02$ at 6 months, $P=0.04$ at 12 months) and according to the efficacy analysis ( $P=0.03$ at 6 months, $P=0.08$ at 12 months) (Table 2)."

Table 2:

"Intention-to-treat population 6 month: $16 / 76$ (21.1\%) vs. $29 / 76$ (38.2\%), OR: 0.43 (0.21-0.89), $p=0.02$ 12 month: $14 / 76$ (18.4\%) vs. 25/76 (32.9\%), OR: 0.46 (0.22-0.98), p=0.04"

Comment: The incomplete outcome data for the 4 to 6 week time point used in our meta-analysis are addressed satisfactorily, albeit with an assumption that favoured HBO. However, the same cannot be said for the 6 and 12 month data which as presented are largely a construct of the assumptions applied rather than based on the actual data (which would have shown there to be very little difference at these time points (i.e. 24 vs 29 abnormal rather than 14 vs 25 )). 
Weaver 2002 (Continued)

Free of selective reporting?
High risk 
Comment: While not easy to work through these long paragraphs it is apparent that the defined thresholds for abnormal results changed on each occasion and that self-reported difficulties with memory, attention and concentration were added to the definition between 1995 and 2001/2.

[Note that given 6 different neuropsychological tests were used it is likely that most people would have either one of these tests $>1$ SD below the mean or 2 tests $>0.5 \mathrm{SD}$ below the mean by chance (regardless of any disease or an influence of treatment). Therefore, the reported differences in PNS between the groups is likely to have been the result of a difference in self-reported symptoms in the 2002 report. This would not have been the case for the definition of PNS described in 1995 because at that time there was no component of the outcome that related to self-reported symptoms].

Comment: Dr Weaver has previously responded to criticisms regarding the apparent change in the outcome definitions, stating that the earlier publications were all inaccurate, and that the only accurate record of outcomes is in the final 2002 report. http://www.cochranefeedback.com/cf/cda/citation.do?id=9531\#9531

Free of other bias? High risk

Power/Sample size/stopping rules:

Quote: "The trial was stopped after the third interim analysis, which included 150 patients, because hyperbaric oxygen was judged to be efficacious $(P<0.01) . "$

Quote: "We calculated that the inclusion of 100 patients in each treatment group would provide the study with a statistical power of 80 percent to detect the difference between the published rates of cognitive sequelae of 5.8 percent and 18.5 percent (with a two-sided probability of a type I error of 0.05 ). The Statistical Data Center at LDS Hospital planned and performed interim analyses after the six-week data were available from 50, 100, and 150 enrolled patients, using a stopping rule requiring a two-sided $P$ value of $0.0001,0.001$, and

0.01 , respectively, for the three analyses. The statisticians and investigators, who were blinded to patients' treatment-group assignments, had access to the results of the interim analyses."

Quote: "For all 152 patients in the intention-to-treat population, cognitive sequelae at six weeks were less frequent in the hyperbaric-oxygen group (25.0 percent) than in the normobaric-oxygen group (46.1 percent; unadjusted odds ratio, 0.39 [95 percent confidence interval, 0.20 to 0.78 ]; $P=0.007$ ) (Table 2). The same was true for the 147 patients with complete data on neuropsychological tests at six weeks: cognitive sequelae were less frequent at six weeks among patients who received hyperbaric-oxygen treatment (24.0 percent) than among those who received normobaric oxygen (43.1 percent, $\mathrm{P}=0.01$ )".

Comment: Note the assumption that those with missing data had neurological sequelae discussed above brought the interim analysis $p$ value to $<0.01$ (i.e. the trial would not have stopped if this assumption hadn't been applied).

In any case, premature stopping of small trials "for benefit" inevitably greatly exaggerates the observed effect for statistical reasons (Bassler 2010).

\section{Characteristics of excluded studies [ordered by study ID]}




\begin{tabular}{ll}
\hline Study & Reason for exclusion \\
\hline Ducasse 1995 & $\begin{array}{l}\text { Surrogate outcomes (EEG, cerebral blood flow reactivity to acetazolamide) examined rather than } \\
\text { symptoms of DNS }\end{array}$ \\
\hline Gao 2002 & Compared HBO vs. HBO plus an antiplatelet agent \\
\hline Hampson 2006 & $\begin{array}{l}\text { Compared HBO at 2.4 ATM vs. HBO by the US Air Force CO protocol (3.0 ATM abs maximum pres- } \\
\text { sure) }\end{array}$ \\
\hline Hopkins 2007 & Post hoc analysis of a genotype tested in 86/152 of the patients in Weaver 2002 \\
\hline Weaver 2007 & Ongoing study comparing one vs three treatments of HBO \\
\hline
\end{tabular}

DATA AND ANALYSES

Comparison 1. Hyperbaric Oxygen (HBO) vs. Normobaric Oxygen (NBO)

\begin{tabular}{llllll}
\hline Outcome or subgroup title & $\begin{array}{l}\text { No. of } \\
\text { studies }\end{array}$ & $\begin{array}{l}\text { No. of } \\
\text { partici- } \\
\text { pants }\end{array}$ & Statistical method & Effect size \\
\hline $\begin{array}{l}1 \text { Presence of symptoms or signs at time of pri- } \\
\text { mary analysis (4-6 weeks) }\end{array}$ & 6 & 1361 & $\begin{array}{l}\text { Odds Ratio (M-H, Random, 95\% } \\
\mathrm{Cl})\end{array}$ & $0.78[0.54,1.12]$ \\
\hline $\begin{array}{l}1.1 \text { Presence of signs or symptoms } \\
\text { nat }\end{array}$ & 6 & 1361 & $\begin{array}{l}\text { Odds Ratio (M-H, Random, 95\% } \\
\mathrm{Cl})\end{array}$ & $0.78[0.54,1.12]$ \\
\hline
\end{tabular}

Analysis 1.1. Comparison 1 Hyperbaric Oxygen (HBO) vs. Normobaric Oxygen (NBO), Outcome 1 Presence of symptoms or signs at time of primary analysis (4-6 weeks).

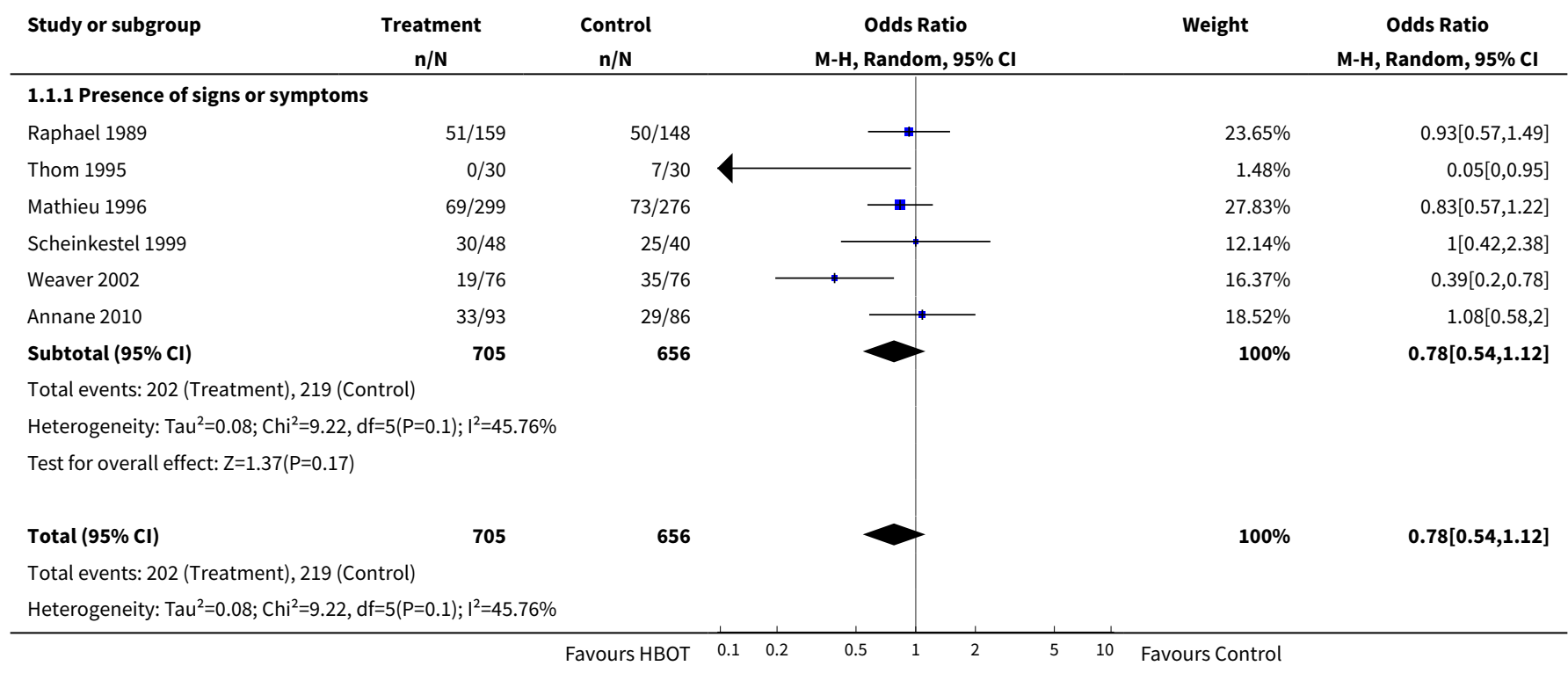




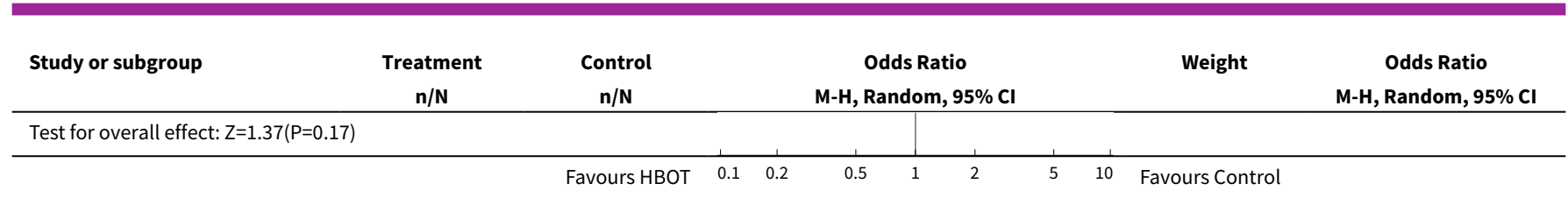

\section{APPENDICES}

\section{Appendix 1. Search strategy}

\section{Search strategies: June 2010}

Cochrane Injuries Group Specialised Register (searched 07 June 2010)

((Hyperbaric and Oxygen") or HBO or HBOT) and ("carbon monoxide" or monoxide* or CO)

\section{The Cochrane Library 2010, Issue 2}

\#1 MeSH descriptor Carbon Monoxide Poisoning explode all trees

\#2 carbon monoxide near3 poison*

\#3 illuminating gas near3 poison*

\#4 carbon monoxide near3 intoxicat*

\#5 Toxic near3 asphyxiation

\#6 monoxide* or $\mathrm{CO}$

\#7 (\#1 OR \#2 OR \#3 OR \#4 OR \#5 OR \#6)

\#8 MeSH descriptor Hyperbaric Oxygenation explode all trees

\#9 high next pressure next oxygen*

\#10 High next tension next oxygen*

\#11 HBO or HBOT

\#12 Hyperbaric next Oxygen*

\#13 multiplace near3 chamber*

\#14 monoplace near3 chamber*

\#15 (\#8 OR \#9 OR \#10 OR \#11 OR \#12 OR \#13 OR \#14)

\#16 (\#7 AND \#15)

\section{MEDLINE (Ovid) 1950 to May Week 42010}

1. exp Carbon Monoxide Poisoning/

2. (carbon monoxide adj3 poison*).ab,ti.

3. (illuminating gas adj3 poison $\left.{ }^{\star}\right)$.ab,ti.

4. (carbon monoxide adj3 intoxicat $\left.{ }^{\star}\right)$.ab,ti.

5. Toxic asphyxiation.ab,ti.

6. (monoxide* or $\mathrm{CO})$.ab,ti.

7. 1 or 2 or 3 or 4 or 5 or 6

8. exp Hyperbaric Oxygenation/

9. (high adj3 (pressure or tension) adj3 oxygen ${ }^{\star}$ ).ab,ti.

10. oxygen ${ }^{\star}$.ti.

11. (high adj3 (pressure or tension)).ab,ti.

12. 10 and 11

13. (HBO or HBOT).ab,ti.

14. ((multiplace or monoplace) adj3 chamber $\left.{ }^{\star}\right) \cdot a b, t i$.

15. multiplace chamber ${ }^{\star}$.ab,ti.

16. monoplace chamber*ab,ti.

17. Hyperbaric Oxygen ${ }^{\star}$.ab,ti.

18.8 or 9 or 12 or 13 or 14 or 15 or 16 or 17

19. randomi?ed.ab,ti.

20. randomised controlled trial.pt.

21. controlled clinical trial.pt.

22. placebo.ab.

23. clinical trials as topic.sh.

24. randomly.ab.

25. trial.ti.

Hyperbaric oxygen for carbon monoxide poisoning (Review) 
26. 19 or 20 or 21 or 22 or 23 or 24 or 25

27. (animals not (humans and animals)).sh.

28. 26 not 27

29. 7 and 18 and 28

\section{EMBASE 1980 to Week 22 (June) 2010}

1. exp Hyperbaric Oxygen/

2. (high adj3 (pressure or tension) adj3 oxygen`).ab,ti.

3. oxygen ${ }^{\star}$.ti.

4. (high adj3 (pressure or tension)).ab,ti.

5. 3 and 4

6. (HBO or HBOT).ab,ti.

7. ((multiplace or monoplace) adj3 chamber $\left.{ }^{\star}\right)$.ab,ti.

8. multiplace chamber*.ab,ti.

9. monoplace chamber ${ }^{\star}$.ab,ti.

10. Hyperbaric Oxygen ${ }^{\star}$.ab,ti.

11. 1 or 2 or 5 or 6 or 7 or 8 or 9 or 10

12. exp carbon monoxide intoxication/

13. (carbon monoxide adj3 poison^).ab,ti.

14. (carbon monoxide adj3 intoxicat $\left.{ }^{\star}\right)$.ab,ti.

15. (illuminating gas adj3 poison ${ }^{\star}$ ).ab,ti.

16. (Toxic adj3 asphyxiat ${ }^{\star}$ ).ab,ti.

17. (monoxide* or CO).ab,ti.

18. 12 or 13 or 14 or 15 or 16 or 17

19. exp Randomized Controlled Trial/

20. exp controlled clinical trial/

21. exp controlled study/

22. randomi?ed.ab,ti.

23. placebo.ab.

24. ${ }^{\star}$ Clinical Trial/

25. exp major clinical study/

26. randomly.ab.

27. (trial or study).ti.

28. 19 or 20 or 21 or 22 or 23 or 24 or 25 or 26 or 27

29. exp animal/ not (exp human/ and exp animal/)

30.28 not 29

31.11 and 18 and 30

PubMed [www.ncbi.nlm.nih.gov/sites/entrez/] (searched 9 June 2010; added to PubMed in the last 5 years)

1. "Carbon Monoxide Poisoning"[Mesh]

2. carbon monoxide AND poison ${ }^{\star}$

3. 1 or 2

4. Hyperbaric Oxygen*

5. $\mathrm{HBO}$ or HBOT

6.4 or 5

7. 3 and 6 (Limits: Clinical Trial, Randomized Controlled Trial, Clinical Trial, Phase I, Clinical Trial, Phase II, Clinical Trial, Phase III, Clinical Trial, Phase IV, Comparative Study, Controlled Clinical Trial, Evaluation Studies, Multicenter Study, published in the last 5 years)

ISI Web of Science: Science Citation Index Expanded (SCI-EXPANDED) (1970 to June 2010), ISI Web of Science: Conference Proceedings Citation Index-Science (CPCI-S) (1990 to June 2010)

Topic $=\left(\left(\right.\right.$ carbon monoxide or monoxide ${ }^{\star}$ or CO) same poison $\left.{ }^{\star}\right)$ AND Topic=((monoplace same chamber $\left.{ }^{\star}\right)$ or $\left(\right.$ multiplace same chamber $\left.^{\star}\right)$ or (hyperbaric same oxygen*) or (high tension same oxygen*) or (high pressure same oxygen*) or HBO or HBOT) AND Topic=((clinical OR control* OR placebo OR random OR randomised OR randomised OR randomly OR random order OR random sequence OR random allocation OR randomly allocated OR at random) SAME (trial ${ }^{\star}$ or group* or study or studies or placebo or controlled))

\section{Global Health 1910 to May 2010}

1.(carbon monoxide adj3 poison $\left.{ }^{\star}\right)$.ab,ti.

2.(illuminating gas adj3 poison*).ab,ti.

3. (carbon monoxide adj3 intoxicat $\left.{ }^{\star}\right)$.ab,ti.

4.(monoxide* or $\mathrm{CO})$. ab,ti.

5.1 or 2 or 3 or 4

6.(high adj3 (pressure or tension) adj3 oxygen $\left.{ }^{\star}\right)$.ab,ti.

Hyperbaric oxygen for carbon monoxide poisoning (Review) 
7.oxygen ${ }^{\star}$. ti.

8.(high adj3 (pressure or tension)).ab,ti.

9.7 and 8

10.(HBO or HBOT).ab,ti.

11.((multiplace or monoplace) adj3 chamber $\left.{ }^{\star}\right)$.ab,ti.

12. multiplace chamber*.ab,ti.

13.monoplace chamber*.ab,ti.

14. Hyperbaric Oxygen ${ }^{\star}$.ab,ti.

15.6 or 9 or 10 or 11 or 12 or 13 or 14

16.5 and 15

\section{WHAT'S NEW}

\begin{tabular}{lll}
\hline Date & Event & Description \\
\hline 17 February 2011 & $\begin{array}{l}\text { New citation required but conclusions } \\
\text { have not changed }\end{array}$ & $\begin{array}{l}\text { Complete revision. Search updated June 2010. No new trials } \\
\text { identified; new publications found including the final publica- } \\
\text { tion of a trial only published in abstract at the time of previous } \\
\text { review. Risk of bias and summary of findings tables added as per } \\
\text { revised Cochrane guidance documents. The authors of the re- } \\
\text { view have changed. }\end{array}$ \\
& \\
\hline
\end{tabular}

\section{HIST ORY}

Protocol first published: Issue 2, 2000

Review first published: Issue 2, 2000

\begin{tabular}{lll}
\hline Date & Event & Description \\
\hline 11 September 2008 & Amended & Converted to new review format. \\
\hline 3 November 2004 & New search has been performed & $\begin{array}{l}\text { The search was updated in October 2004. Two new trials have } \\
\text { been added (Weaver 2002, Raphael 2004), although the latter } \\
\text { is published only in abstract form. The discussion section has } \\
\text { been revised accordingly, and now includes a critical overview of } \\
\text { some of the methodologic and statistical limitations presented } \\
\text { by each of the published trials. }\end{array}$ \\
\hline
\end{tabular}

\section{CONTRIBUTIONS OF AUTHORS}

In the 2005 version, DJ screened citations for eligibility, obtained references, contacted authors, extracted data, entered data and wrote the review. NB, MS, and MB screened citations for eligibility, extracted data and helped to write the review. GI and MM obtained references and helped to write the review.

In this 2010 version, NB screened citations for eligibility, obtained references, contacted authors, extracted data, entered data, undertook the GRADE evaluation and wrote the review. EL and MB screened citations for eligibility, extracted \& rated data and helped to write the review. GI and DJ undertook the GRADE evaluation creating the summary of findings table and helped to write the review.

\section{DECLARATIONS OF INTEREST}

NB, DJ, GI, MB: None known.

EL: From 2000 - 2002 and 2006 - 2008, Dr. Lavonas served as medical director of the hyperbaric medicine unit at Carolinas Medical Center, Charlotte, North Carolina, USA. Dr. Lavonas left that institution in 2008, and the emergency hyperbaric unit was closed in 2009. Dr. Lavonas reports no current competing interests involving the subject of this review. 


\section{SOURCES OF SUPPORT}

\section{Internal sources}

- No sources of support supplied

\section{External sources}

- Canadian Institutes for Health Research (CIHR), Canada.

\section{DIFFERENCES BETWEEN PROTOCOLAND REVIEW}

The methods have been updated since publication of the protocol to reflect revised guidance from The Cochrane Collaboration (Higgins 2008). Thus we used risk of bias assessment rather than the Jadad scale and Schulz assessment of allocation concealment. We also incorporated a GRADE summary of findings assessment. These changes in methods have not altered the overall conclusions about the strength of evidence from the 2005 version.

\section{NOTES}

In the next update to the review we will include studies comparing different protocols for HBO treatment for CO poisoning - for example: one HBO treatment session vs. two or more, 2ATA HBO vs. 3 ATA HBO, etc....

\section{INDEX TERMS}

\section{Medical Subject Headings (MeSH)}

*Hyperbaric Oxygenation; Carbon Monoxide Poisoning [complications] [*therapy]; Nervous System Diseases [prevention \& control]; Oxygen Inhalation Therapy; Randomized Controlled Trials as Topic

\section{MeSH check words}

Humans 